\title{
Twisted Spectral Triples and Connes' Character Formula
}

\author{
Farzad Fathizadeh \\ Department of Mathematics and Statistics \\ York University \\ Toronto, Ontario, Canada, M3J 1P3 \\ ffathiza@mathstat.yorku.ca \\ Masoud Khalkhali \\ Department of Mathematics \\ The University of Western Ontario \\ London, Ontario, Canada, N6A 5B7 \\ masoud@uwo.ca
}

\begin{abstract}
We give a proof of an analogue of Connes' Hochschild character theorem for twisted spectral triples obtained from twisting a spectral triple by scaling automorphisms, under some suitable conditions. We also survey some of the properties of twisted spectral triples that are known so far.
\end{abstract}

\section{Introduction}

In 8. Connes and Moscovici introduced a refinement of the notion of spectral triple called twisted spectral triple. As is well known by now, the metric aspects of a geometric space in noncommutative geometry is encoded by a spectral triple 5. This concept however puts rather severe restrictions on the underlying noncommutative space, as it forces the existence of a tracial state. In particular it is inapplicable in type III situations according to Murray-von Neumann's classification of von Neumann algebras into types. It was to deal with these type III examples that twisted spectral triples were introduced.

In a twisted spectral triple, the twist is afforded by an automorphism. More precisely, there is an automorphism $\sigma$ of the underlying noncommutative space $\mathcal{A}$ such that all twisted commutators $[D, a]_{\sigma}=D a-\sigma(a) D$ are bounded operators ( $D$ is a selfadjoint operator playing the role of the Dirac operator). While there are many natural examples of twisted spectral triples, proving general results about them, parallel to the untwisted case, have proved to be a challenging task. The most celebrated among such questions are perhaps the extension of Connes' 
character formula for the Hochschild class of the Connes-Chern character of a spectral triple [5, as well as an extension of Connes-Moscovici's local index formula, 7] to twisted spectral triples. In this paper we give a proof of a character formula for twisted spectral triples obtained from twisting a spectral triple by scaling automorphisms, under suitable conditions. We shall also briefly outline Moscovici's local index formula for twisted spectral triples obtained by conformal perturbation of a spectral triple [17.

This paper is organized as follows. In Section 2 we recall the basic notions of spectral triples, noncommutative integration and residue functionals for a spectral triple. We also recall Connes and Moscovici's local index formula [7. We then recall the notion of twisted spectral triples [8. In Section 3 we recall several general methods to construct twisted spectral triples from 8], 17] and 11. An important idea here is twisting a spectral triple by scaling automorphisms and the corresponding algebra of twisted pseudodifferential operators introduced by Moscovici [17. In Section 4 we recall some non-obvious properties of twisted spectral triples from [8], among them the fact that one can define a Connes-Chern character for twisted spectral triples with values in ordinary non-twisted cyclic cohomology. Equivalently one can define a pairing between a finitely summable twisted spectral triple and $K$-theory. In section 5 we give a proof of an analogue of Connes' Hochschild character theorem for twisted spectral triples obtained from twisting a spectral triple by scaling automorphisms. Finally in the last section we recall Moscovici's Ansatz and proof of the local index formula for twisted spectral triples obtained by scaling automorphisms 17.

\section{$2 \quad$ From spectral triples to twisted spectral triples}

The notion of a geometric space in noncommutative geometry is encoded by a spectral triple. This concept however puts rather severe restrictions on the underlying noncommutative space that renders it inapplicable in type III situations according to Murray-von Neumann classification of von Neumann algebras into types. We shall first try to explain this point.

We start with a quick review of the Dixmier trace and the noncommutative integral, following closely [5]. Let $\mathcal{H}$ be a Hilbert space and let $\mathcal{K}(\mathcal{H})$ denote the two sided ideal of compact operators on $\mathcal{H}$. For a compact operator $T: \mathcal{H} \rightarrow \mathcal{H}$, let

$$
\mu_{1}(T) \geq \mu_{2}(T) \geq \cdots \geq 0
$$

denote the sequence of eigenvalues of its absolute value $|T|:=\left(T^{*} T\right)^{\frac{1}{2}}$, written in decreasing order. Thus, by the minimax principle, $\mu_{1}(T)=\|T\|$, and in general

$$
\mu_{n}(T)=\inf \left\|\left.T\right|_{V}\right\|, \quad n \geq 1,
$$

where the infimum is over the set of subspaces of codimension $n-1$, and $\left.T\right|_{V}$ denotes the restriction of $T$ to the subspace $V$. A compact operator $T \in \mathcal{K}(\mathcal{H})$ 
is called trace class if $\sum \mu_{n}(T)<\infty$, and in that case the trace of $T$ is defined by

$$
\operatorname{Trace}(T)=\sum_{n}\left(T e_{n}, e_{n}\right)
$$

where $\left\{e_{n}\right\}_{n=1}^{\infty}$ is an orthonormal basis for $\mathcal{H}$. It is known that this is the unique normal trace on the $C^{*}$-algebra $\mathcal{L}(\mathcal{H})$ of bounded operators on $\mathcal{H}$. The question of existence of non-normal traces on $\mathcal{L}(\mathcal{H})$ was left open until it was settled affirmatively by Jacques Dixmier. In [9] Dixmier shows that there are uncountably many non-normal traces on $\mathcal{L}(\mathcal{H})$. Many years later, Alain Connes discovered that these non-normal traces can in fact be used to define a process of noncommutative integration in noncommutative geometry as we describe next.

The natural domain of a Dixmier trace is the set of operators

$$
\mathcal{L}^{1, \infty}(\mathcal{H})=\left\{T \in \mathcal{K}(\mathcal{H}) ; \quad \sum_{1}^{N} \mu_{n}(T)=O(\log N)\right\} .
$$

Notice that trace class operators are automatically in $\mathcal{L}^{1, \infty}(\mathcal{H})$. The Dixmier trace of an operator $T \in \mathcal{L}^{1, \infty}(\mathcal{H})$ measures the logarithmic divergence of its ordinary trace. More precisely, we are interested in the limit of the bounded sequence

$$
\sigma_{N}(T)=\frac{\sum_{1}^{N} \mu_{n}(T)}{\log N}, \quad N=1,2, \ldots
$$

as $N \rightarrow \infty$. The first problem of course is that, while by our assumption the sequence is bounded, the usual limit may not exist and must be replaced by a generalized limit, similar to Banach limits of non-convergent bounded sequences. A more challenging task is to make sure that our generalized limit still defines a trace.

To this end, let $\operatorname{Trace}_{\Lambda}(T), \Lambda \in[1, \infty)$, be the piecewise affine interpolation of the partial trace function $\operatorname{Trace}_{N}(T)=\sum_{1}^{N} \mu_{n}(T)$. Recall that a state on a $C^{*}$-algebra is a non-zero positive linear functional on the algebra. Let $\omega$ : $C_{b}[e, \infty) \rightarrow \mathbb{C}$ be a normalized state on the algebra of bounded continuous functions on $[e, \infty)$ such that $\omega(f)=0$ for all $f$ vanishing at $\infty$. Now, using $\omega$, the Dixmier trace of a positive operator $T \in \mathcal{L}^{1, \infty}(\mathcal{H})$ is defined as

$$
\operatorname{Tr}_{\omega}(T):=\omega\left(\tau_{\Lambda}(T)\right),
$$

where

$$
\tau_{\Lambda}(T)=\frac{1}{\log \Lambda} \int_{e}^{\Lambda} \frac{\operatorname{Trace}_{r}(T)}{\log r} \frac{d r}{r}
$$

is the Cezaro mean of the function $\frac{\operatorname{Trace}_{r}(T)}{\log r}$ over the multiplicative group $\mathbb{R}_{+}^{*}$. One then extends $\operatorname{Tr}_{\omega}$ to all of $\mathcal{L}^{1, \infty}(\mathcal{H})$ by linearity.

The resulting linear functional $\operatorname{Tr}_{\omega}$ is a positive trace on $\mathcal{L}^{1, \infty}(\mathcal{H})$. It is easy to see from its definition that if $T$ actually happens to be a trace class operator then $\operatorname{Tr}_{\omega}(T)=0$ for all $\omega$, i.e., the Dixmier trace is invariant under 
perturbations by trace class operators. This is a very useful property and makes $\operatorname{Tr}_{\omega}$ a flexible tool in computations.

The Dixmier trace, $\operatorname{Tr}_{\omega}$, in general depends on the limiting procedure $\omega$, however, for the class of operators $T$ for which $\operatorname{Lim}_{\Lambda \rightarrow \infty} \tau_{\Lambda}(T)$ exits, it is independent of the choice of $\omega$ and is equal to $\operatorname{Lim}_{\Lambda \rightarrow \infty} \tau_{\Lambda}(T)$. One of the main results proved in [4] is that if $M$ is a closed $n$-dimensional manifold, $E$ is a smooth vector bundle on $M, P$ is a pseudodifferential operator of order $-n$ acting between $L^{2}$-sections of $\mathrm{E}$, and $\mathcal{H}=L^{2}(M, E)$, then $P \in \mathcal{L}^{1, \infty}(\mathcal{H})$ and, for any choice of $\omega, \operatorname{Tr}_{\omega}(P)=n^{-1} \operatorname{Res}(P)$. Here Res denotes Wodzicki's noncommutative residue [19, 15]. For example, if $D$ is an elliptic first order differential operator, $|D|^{-n}$ is a pseudodifferential operator of order $-n$, and the Dixmier trace $\operatorname{Tr}_{\omega}\left(|D|^{-n}\right)$ is independent of the choice of $\omega$.

Next, we would like to explain the notion of spectral triple. This concept has its roots in $K$-homology and Riemannian geometry simultaneously. We start by explaining the notion of a Fredholm module which is the conformal counterpart of a spectral triple.

An odd Fredholm module over a unital algebra $\mathcal{A}$ is a pair $(\mathcal{H}, F)$ where $\mathcal{H}$ is a Hilbert space on which the algebra $\mathcal{A}$ acts by bounded operators and $F \in \mathcal{L}(\mathcal{H})$ is a selfadjoint operator such that $F^{2}=i d$, and such that the commutators $[F, \pi(a)]$ are compact operators for all $a \in \mathcal{A}$. Here $\pi: \mathcal{A} \rightarrow \mathcal{L}(\mathcal{H})$ denotes a unital action of $\mathcal{A}$ on $\mathcal{H}$. A Fredholm module is called $p$-summable $(1 \leq p<\infty)$, if $[F, \pi(a)] \in \mathcal{L}^{p}(\mathcal{H})$ for all $a \in \mathcal{A}$, where $\mathcal{L}^{p}(\mathcal{H})$ is the Schatten ideal of $p$ summable compact operators 3 . Fredholm modules should be thought of as representing $K$-homology classes defined by abstract elliptic partial differential operators on the noncommutative space $\mathcal{A}$.

Spectral triples provide a refinement of Fredholm modules. Going from Fredholm modules to spectral triples is akin to going from the conformal class of a Riemannian metric to the metric itself. Spectral triples simultaneously provide a notion of Dirac operator in noncommutative geometry, as well as a Riemannian type distance function for noncommutative spaces as we shall explain next.

To motivate the definition of a spectral triple, we recall that the Dirac operator $D$ on a compact Riemannian $\operatorname{spin}^{c}$ manifold acts as an unbounded selfadjoint operator on the Hilbert space $L^{2}(M, S)$ of $L^{2}$-spinors on the manifold $M$. If we let $C^{\infty}(M)$ act on $L^{2}(M, S)$ by multiplication operators, then one can check that for any smooth function $f$, the commutator $[D, f]=D f-f D$ extends to a bounded operator on $L^{2}(M, S)$. Now the geodesic distance $d$ on $M$ can be recovered from the following beautiful distance formula of Connes [5]:

$$
d(p, q)=\operatorname{Sup}\{|f(p)-f(q)| ; \quad\|[D, f]\| \leq 1\}, \quad \forall p, q \in M .
$$

The triple $\left(C^{\infty}(M), L^{2}(M, S), D\right)$ is a commutative example of a spectral triple. Its general definition, in the odd case, is as follows.

Definition 2.1. Let $\mathcal{A}$ be a unital algebra. An odd spectral triple on $\mathcal{A}$ is a triple $(\mathcal{A}, \mathcal{H}, D)$ consisting of a Hilbert space $\mathcal{H}$, a selfadjoint unbounded operator $D$ : $\operatorname{Dom}(D) \subset \mathcal{H} \rightarrow \mathcal{H}$ with compact resolvent, i.e., $(D+\lambda)^{-1} \in \mathcal{K}(\mathcal{H})$ for all $\lambda \notin \mathbb{R}$, 
and a unital representation $\pi: \mathcal{A} \rightarrow \mathcal{L}(\mathcal{H})$ of $\mathcal{A}$ such that for all a $\in \mathcal{A}$, the commutator $[D, \pi(a)]$ is defined on $\operatorname{Dom}(D)$ and extends to a bounded operator on $\mathcal{H}$.

The finite summability assumption for Fredholm modules has a finer analogue for spectral triples. For simplicity we shall assume that $D$ is invertible (in general, since $\operatorname{Ker}(D)$ is finite dimensional, by restricting to its orthogonal complement we can always reduce to this case). A spectral triple is called finitely summable if for some $n \geq 1$,

$$
|D|^{-n} \in \mathcal{L}^{1, \infty}(\mathcal{H}) .
$$

A simple example of an odd spectral triple is $\left(C^{\infty}\left(S^{1}\right), L^{2}\left(S^{1}\right), D\right)$, where $S^{1}=\mathbb{R} / 2 \pi \mathbb{Z}$ is the circle and $D$ is the unique selfadjoint extension of the operator $\frac{1}{i} \frac{d}{d x}$. The eigenvalues of $|D|$ are $|n| ; n \in \mathbb{Z}$ which shows that if we restrict $D$ to the orthogonal compliment of constant functions then $|D|^{-1} \in$ $\mathcal{L}^{1, \infty}\left(L^{2}\left(S^{1}\right)\right)$.

Given a spectral triple $(\mathcal{A}, \mathcal{H}, D)$, one can obtain a Fredholm module $(\mathcal{A}, \mathcal{H}, F)$ by choosing $F=\operatorname{Sign}(D)=D|D|^{-1}$. Connes' Hochschild character formula gives a local expression for the Hochschild class of the Connes-Chern character of $(\mathcal{A}, \mathcal{H}, F)$ in terms of $D$ itself. For this one has to assume that the spectral triple $(\mathcal{A}, \mathcal{H}, D)$ is regular in the sense that for all $a \in \mathcal{A}$,

$$
a \text { and }[D, a] \in \cap \operatorname{Dom}\left(\delta^{k}\right)
$$

where the derivation $\delta$ is given by $\delta(x)=[|D|, x]$.

Now, assuming (2.1) holds, Connes defines an $(n+1)$-linear functional $\varphi$ on $\mathcal{A}$ by

$$
\varphi\left(a^{0}, a^{1}, \ldots, a^{n}\right)=\operatorname{Tr}_{\omega}\left(a^{0}\left[D, a^{1}\right] \cdots\left[D, a^{n}\right]|D|^{-n}\right) .
$$

It can be shown that $\varphi$ is a Hochschild $n$-cocycle on $\mathcal{A}$. We recall that a Hochschild $n$-cycle $c \in Z_{n}(A, A)$ is an element $c=\sum a^{0} \otimes a^{1} \otimes \cdots \otimes a^{n} \in A^{\otimes(n+1)}$ such that its Hochschild boundary $b(c)=0$, where $b$ is defined by (5.4). The following result, known as Connes' Hochschild character formula, computes the Hochschild class of the Chern character by a local formula, i.e., in terms of $\varphi$ :

Theorem 2.1. [5] Let $(\mathcal{A}, \mathcal{H}, D)$ be a regular spectral triple. Let $F=\operatorname{Sign}(D)$ denote the sign of $D$ and $\tau_{n} \in H C^{n}(\mathcal{A})$ denote the Connes-Chern character of $(\mathcal{H}, F)$. For every $n$-dimensional Hochschild cycle $c=\sum a^{0} \otimes a^{1} \otimes \cdots \otimes a^{n} \in$ $Z_{n}(\mathcal{A}, \mathcal{A})$, one has

$$
\left\langle\tau_{n}, c\right\rangle=\sum \varphi\left(a^{0}, a^{1}, \ldots, a^{n}\right) .
$$

Identifying the full cyclic cohomology class of the Connes-Chern character of $(\mathcal{A}, \mathcal{H}, D)$ by a local formula is the content of Connes-Moscovici's local index formula. For this we have to assume the spectral triple satisfies another technical condition. Let $\mathcal{B}$ denote the subalgebra of $\mathcal{L}(\mathcal{H})$ generated by operators $\delta^{k}(a)$ and $\delta^{k}([D, a]), k \geq 1$. A spectral triple is said to have a discrete dimension spectrum $\Sigma$ if $\Sigma \subset \mathbb{C}$ is discrete and for any $b \in \mathcal{B}$ the function

$$
\zeta_{b}(z)=\operatorname{Trace}\left(b|D|^{-z}\right), \quad \operatorname{Re} z>n,
$$


extends to a holomorphic function on $\mathbb{C} \backslash \Sigma$. It is further assumed that $\Sigma$ is simple in the sense that $\zeta_{b}(z)$ has only simple poles in $\Sigma$.

The local index formula of Connes and Moscovici [7] is given by the following theorem (we have used the formulation in [6]):

Theorem 2.2. [6] 1. The equality

$$
f P=\operatorname{Res}_{z=0} \operatorname{Trace}\left(P|D|^{-z}\right)
$$

defines a trace on the algebra generated by $\mathcal{A},[D, \mathcal{A}]$, and $|D|^{z}, z \in \mathbb{C}$.

2. There are only a finite number of non-zero terms in the following formula which defines the odd components $\left(\varphi_{n}\right)_{n=1,3, \ldots}$ of an odd cyclic cocycle in the $(b, B)$ bicomplex of $\mathcal{A}$ : For each odd integer $n$ let

$$
\varphi_{n}\left(a^{0}, \cdots, a^{n}\right):=\sum_{k} c_{n, k} f a^{0}\left[D, a^{1}\right]^{\left(k_{1}\right)} \ldots\left[D, a^{n}\right]^{\left(k_{n}\right)}|D|^{-n-2|k|}
$$

where $T^{(k)}:=\nabla^{k}$ and $\nabla(T)=D^{2} T-T D^{2}, k$ is a multi-index, $|k|=k_{1}+\cdots+k_{n}$ and

$$
c_{n, k}:=(-1)^{|k|} \sqrt{2 i}\left(k_{1} ! \ldots k_{n} !\right)^{-1}\left(\left(k_{1}+1\right) \cdots\left(k_{1}+k_{2}+\cdots k_{n}\right)\right)^{-1} \Gamma\left(|k|+\frac{n}{2}\right) .
$$

3. The pairing of the cyclic cohomology class $\left(\varphi_{n}\right) \in H C^{*}(\mathcal{A})$ with $K_{1}(\mathcal{A})$ gives the Fredholm index of $D$ with coefficients in $K_{1}(\mathcal{A})$.

Given an $n$-summable regular spectral triple $(\mathcal{A}, \mathcal{H}, D)$, the linear functional

$$
a \mapsto \operatorname{Tr}_{\omega}\left(a|D|^{-n}\right)
$$

defines a trace on the algebra $\mathcal{A}$ ( $c f$. Proposition 4.1). Thus to deal with type III algebras which carry no non-trivial traces, the notion of spectral triple must be modified. In 8 . Connes and Moscovici define a notion of twisted spectral triple, where the twist is afforded by an algebra automorphism (related to the modular automorphism group). More precisely, one postulates that there exists an automorphism $\sigma$ of $\mathcal{A}$ such that the twisted commutators $D a-\sigma(a) D$ are bounded operators for all $a \in \mathcal{A}$. Here is the full definition:

Definition 2.1. Let $\mathcal{A}$ be an algebra which is represented by bounded operators in a Hilbert space $\mathcal{H}$, and $D$ be an unbounded selfadjoint operator in $\mathcal{H}$ with compact resolvent. With $\sigma$ being an automorphism of $\mathcal{A}$, the triple $(\mathcal{A}, \mathcal{H}, D)$ is called a twisted spectral triple or a $\sigma$-spectral triple if for any $a \in \mathcal{A}$, the twisted commutator

$$
[D, a]_{\sigma}:=D a-\sigma(a) D
$$

is defined on the domain of $D$, and extends to a bounded operator in $\mathcal{H}$.

A twisted spectral triple is said to be Lipschitz-regular if the twisted commutators $|D| a-\sigma(a)|D|$ are bounded as well for all $a \in \mathcal{A}$.

A graded twisted spectral triple is one that is endowed with a grading operator $\gamma=\gamma^{*} \in \mathcal{L}(\mathcal{H})$ such that $\gamma^{2}=i d, \gamma$ commutes with the action of $\mathcal{A}$, and anticommutes with $D$. 
When the algebra $\mathcal{A}$ is involutive, the representation is assumed to be involutive as well. In this case, it is natural to impose the following compatibility condition between the automorphism and the involution:

$$
\sigma(a)^{*}=\sigma^{-1}\left(a^{*}\right), \quad \forall a \in \mathcal{A} .
$$

It is shown in 8 that in the twisted case, the Dixmier trace induces a twisted trace on the algebra $\mathcal{A}$, but surprisingly, under some regularity conditions, the Connes-Chern character of the phase space lands in ordinary cyclic cohomology. Thus its pairing with ordinary $K$-theory makes sense, and it can be recovered as the index of Fredholm operators. This suggests the significance of developing a local index formula for twisted spectral triples, i.e. finding a formula for a cocycle, cohomologous to the Connes-Chern character in the $(b, B)$-bicomplex, which is given in terms of twisted commutators and residue functionals. We believe that this new theme of twisted spectral triples, and type III noncommutative geometry in general, shall dominate the subject in near future.

For example, very recently a local index formula has been proved for a class of twisted spectral triples by Henri Moscovici [17. We will discuss this result in detail in Section 6 of this paper. This class is obtained by twisting an ordinary spectral triple $(\mathcal{A}, \mathcal{H}, D)$ by a subgroup $G$ of scaling automorphisms of the triple, i.e. the set of all unitary operators $U \in \mathcal{U}(\mathcal{H})$ such that $U \mathcal{A} U^{*}=\mathcal{A}$, and $U D U^{*}=\mu(U) D$, with $\mu(U)>0$. It is shown that the crossed product algebra $\mathcal{A} \rtimes G$ admits an automorphism $\sigma$, given by the formula $\sigma(a U)=\mu(U)^{-1} a U$, for all $a \in \mathcal{A}, U \in G$; and $(\mathcal{A} \rtimes G, \mathcal{H}, D)$ is a twisted spectral triple.

\section{$3 \quad$ Examples of twisted spectral triples}

In this section, we recall general methods to construct twisted spectral triples.

\subsection{Perturbing spectral triples}

In $[8$, it is shown that starting from an ordinary spectral triple $(\mathcal{A}, \mathcal{H}, D)$ and a selfadjoint element $h=h^{*} \in \mathcal{A}$, the perturbed triple

$$
\left(\mathcal{A}, \mathcal{H}, D^{\prime}\right), D^{\prime}=e^{h} D e^{h}
$$

is a $\sigma$-spectral triple, where the automorphism $\sigma$ is given by

$$
\sigma(a)=e^{2 h} a e^{-2 h}, a \in \mathcal{A} .
$$

In fact for any $a \in \mathcal{A}$, one has

$$
D^{\prime} a-\sigma(a) D^{\prime}=e^{h} D e^{h} a-e^{2 h} a e^{-2 h} e^{h} D e^{h}=e^{h}\left[D, e^{h} a e^{-h}\right] e^{h} .
$$

Therefore the twisted commutators $\left[D^{\prime}, a\right]_{\sigma}$ are bounded since the commutators $[D, b]$ are bounded operators for all $b \in \mathcal{A}$. A concrete example of this construction is obtained when one compares the Dirac operators of conformally equivalent Riemannian metrics as follows: 
Example 3.1. Let $(M, g)$ be a compact Riemannian spin manifold and $D=$ $D^{g}$ be the associated Dirac operator acting on the Hilbert space of $L^{2}$-spinors $\mathcal{H}=L^{2}\left(M, S^{g}\right)$. Let $g^{\prime}=e^{-4 h} g$ be a conformally equivalent metric where $h \in C^{\infty}(M)$ is a selfadjoint element. It can be shown that the gauge transform operator ${ }^{g} D^{g^{\prime}}=\beta_{g}^{g^{\prime}} \circ D^{g^{\prime}} \circ \beta_{g^{\prime}}^{g}$ has the form

$$
{ }^{g} D^{g^{\prime}}=e^{(n+1) h} \circ D^{g} \circ e^{-(n-1) h} .
$$

After the canonical identification of the space of $g$-spinors with $g^{\prime}$-spinors, it can be seen 8 that the gauge transform spectral triple is obtained from the original one by replacing $D$ with

$$
D^{\prime}=e^{h} D e^{h} .
$$

More generally, one can start from a $\sigma$-spectral triple $(\mathcal{A}, \mathcal{H}, D)$ and a selfadjoint element $h=h^{*} \in \mathcal{A}$, and investigate the properties of the perturbed triple

$$
\left(\mathcal{A}, \mathcal{H}, D^{\prime}\right), \quad D^{\prime}=e^{h} D e^{h} .
$$

Lemma 3.2. Let $(\mathcal{A}, \mathcal{H}, D)$ be a $\sigma$-spectral triple, and $h=h^{*} \in \mathcal{A}$. Then the perturbed triple

$$
\left(\mathcal{A}, \mathcal{H}, D^{\prime}\right), \quad D^{\prime}=e^{h} D e^{h}
$$

is a $\sigma^{\prime}$-spectral triple where $\sigma^{\prime} \in A u t(\mathcal{A})$ is given by

$$
\sigma^{\prime}(a)=e^{h} \sigma\left(e^{h} a e^{-h}\right) e^{-h}, a \in \mathcal{A} .
$$

Proof. For any $a \in \mathcal{A}$, one has

$$
D^{\prime} a-\sigma^{\prime}(a) D^{\prime}=e^{h} D e^{h} a-e^{h} \sigma\left(e^{h} a e^{-h}\right) e^{-h} e^{h} D e^{h}=e^{h}\left[D, e^{h} a e^{-h}\right]_{\sigma} e^{h} .
$$

Therefore the twisted commutators $\left[D^{\prime}, a\right]_{\sigma^{\prime}}$ are bounded since the twisted commutators $[D, b]_{\sigma}$ are bounded operators for all $b \in \mathcal{A}$.

\subsection{Twisted connections on crossed product algebras}

In [1], a method for constructing twisted spectral triples on crossed product algebras is suggested. This method uses 1-cocycles to construct automorphisms for crossed product algebras, and explains how one can obtain twisted traces, twisted derivatives, and twisted connections on these algebras. We shall explain this method and show that it can reconstruct an example of twisted spectral triples, first given by Connes and Moscovici in [8]. First we recall some definitions:

Definition 3.3. Let $\sigma: \mathcal{A} \rightarrow \mathcal{A}$ be an automorphism of an algebra $\mathcal{A}$.

1. A $\sigma$-derivation on $\mathcal{A}$ is a linear map $\delta: \mathcal{A} \rightarrow \mathcal{A}$ such that

$$
\delta(a b)=\delta(a) b+\sigma(a) \delta(b), \quad \forall a, b \in \mathcal{A} .
$$


2. A $\sigma$-trace on $\mathcal{A}$ is a linear map $\tau: \mathcal{A} \rightarrow \mathbb{C}$ such that

$$
\tau(a b)=\tau(\sigma(b) a), \quad \forall a, b \in \mathcal{A} .
$$

Now, let $A$ be an algebra with a right action of a group $\Gamma$ by automorphisms:

$$
A \times \Gamma \rightarrow A, \quad(a, \gamma) \mapsto a \cdot \gamma .
$$

We consider the algebraic crossed product $A \rtimes \Gamma$ with the standard multiplication:

$$
(a \otimes \gamma)(b \otimes \mu)=(a \cdot \mu) b \otimes \gamma \mu, \quad a, b \in A, \quad \gamma, \mu \in \Gamma .
$$

Let $Z(A)$ denote the center of the algebra $A$, and $A^{*}$ its group of invertible elements.

Definition 3.4. 1. A map $j: \Gamma \rightarrow Z(A) \cap A^{*}$ is a 1-cocycle if

$$
j(\gamma \mu)=((j \gamma) \cdot \mu) j \mu, \quad \forall \gamma, \mu \in \Gamma .
$$

2. Given a map $j: \Gamma \rightarrow A$, a linear functional $\tau: A \rightarrow \mathbb{C}$ is said to have the change of variable property with respect to $j$, if

$$
\tau((a \cdot \gamma) j \gamma)=\tau(a), \quad \forall a \in A, \quad \gamma \in \Gamma .
$$

Notice that (3.2) amounts to saying that $j$ is a (multiplicative) group $1-$ cocycle for $H^{1}\left(\Gamma, Z(A) \cap A^{*}\right)$.

Proposition 3.5. 11] Let $A$ be an algebra with a right action of a group $\Gamma$ by automorphisms, and $j: \Gamma \rightarrow Z(A) \cap A^{*}$ be a 1-cocycle.

1. The map $\sigma: A \rtimes \Gamma \rightarrow A \rtimes \Gamma$ given by

$$
\sigma(a \otimes \gamma)=\left(\left(j \gamma^{-1}\right) \cdot \gamma\right) a \otimes \gamma
$$

is an automorphism.

2. Let $\delta: A \rightarrow A$ be a derivation such that $\delta(a \cdot \gamma)=(\delta(a) \cdot \gamma) j \gamma$ for all $a \in A, \gamma \in \Gamma$. Then for any $s=1,2, \ldots$, the map $\delta_{s}^{\prime}: A \rtimes \Gamma \rightarrow A \rtimes \Gamma$ defined by

$$
\delta_{s}^{\prime}(a \otimes \gamma)=\left(\delta\left(\left(a \cdot \gamma^{-1}\right)\left(j \gamma^{-1}\right)^{s}\right)\left(j \gamma^{-1}\right)^{-s} \otimes 1\right)(1 \otimes \gamma)
$$

is a $\sigma$-derivation on $A \rtimes \Gamma$. Also $\delta_{s}^{\prime} \circ \sigma=\sigma \circ \delta_{s}^{\prime}$ if $\delta \circ j=0$.

3. If $\tau: A \rightarrow \mathbb{C}$ is a trace such that $\tau \circ \delta=0$, then $\tau^{\prime} \circ \delta_{s}^{\prime}=0$ where the linear functional $\tau^{\prime}: A \rtimes \Gamma \rightarrow \mathbb{C}$ is defined by

$$
\tau^{\prime}(a \otimes \gamma)=0 \text { if } \gamma \neq 1, \text { and } \tau^{\prime}(a \otimes 1)=\tau(a) .
$$

Also, if $\tau$ has the change of variable property with respect to $j$, then $\tau^{\prime}$ is a $\sigma$-trace on $A \rtimes \Gamma$. 
Example 3.6. 1. Let $M$ be a smooth oriented manifold and $\omega$ a volume form on $M$. Let $\Gamma=\operatorname{Diff}(M)$ be the group of diffeomorphisms of $M$. The map $j: \Gamma \rightarrow C^{\infty}(M)$ defined by

$$
\gamma^{*}(\omega)=j(\gamma) \omega
$$

is easily seen to be a 1-cocycle.

2. Let $\chi: \Gamma \rightarrow \mathbb{C}^{*}$ be a 1-dimensional character of a group $\Gamma$ which acts on an algebra $A$ by automorphisms, and let $j(\gamma)=\chi(\gamma) 1_{A}$. Then $j$ is a 1-cocycle, and a derivation $\delta: A \rightarrow A$ is compatible with $j$ if and only if $\delta(a \cdot \gamma)=\chi(\gamma) \delta(a) \cdot \gamma$ for any $a \in A$, and $\gamma \in \Gamma$.

Definition 3.7. Let $\mathcal{A}$ be an algebra, $\delta: \mathcal{A} \rightarrow \mathcal{A}$ a derivation, and $E$ a left $\mathcal{A}$-module. A linear map $\nabla: E \rightarrow E$ is said to be a connection if it satisfies the Leibniz rule, i.e.

$$
\nabla(a \xi)=\delta(a) \xi+a \nabla(\xi), \quad \forall a \in \mathcal{A}, \quad \xi \in E .
$$

If $\sigma: \mathcal{A} \rightarrow \mathcal{A}$ is an automorphism and $\delta: \mathcal{A} \rightarrow \mathcal{A}$ a $\sigma$-derivation, then a linear map $\nabla: E \rightarrow E$ is said to be a twisted connection if it satisfies the twisted Leibniz rule:

$$
\nabla(a \xi)=\delta(a) \xi+\sigma(a) \nabla(\xi), \quad \forall a \in \mathcal{A}, \quad \xi \in E .
$$

This notion of twisted connection comes from [18].

Given a twisted connection $\nabla$, one can try to define a twisted spectral triple by letting $D=\nabla$. Then $\nabla a-\sigma(a) \nabla=\delta(a)$ shows the boundedness condition is satisfied provided $\delta(a)$ acts by a bounded operator.

In the following proposition, $A$ is an algebra endowed with a right action of a group $\Gamma$ by automorphisms, with representations $\pi: A \rightarrow \operatorname{End}(E)$, and $\rho: \Gamma \rightarrow \mathrm{GL}(E)$, defining a covariant system, i.e.

$$
\pi(a \cdot \gamma)=\rho\left(\gamma^{-1}\right) \pi(a) \rho(\gamma), \quad \forall a \in A, \gamma \in \Gamma .
$$

Then we obtain a representation $\pi^{\prime}: A \rtimes \Gamma \rightarrow \operatorname{End}(E)$ given by

$$
\pi^{\prime}(a \otimes \gamma)=\rho(\gamma) \pi(a), \quad \forall a \in A, \gamma \in \Gamma .
$$

Also let $j: \Gamma \rightarrow Z(A) \cap A^{*}$ be a 1-cocycle and $\delta: A \rightarrow A$ a derivation as in Proposition 3.5. Therefore we have an automorphism $\sigma: A \rtimes \Gamma \rightarrow A \rtimes \Gamma$ and we fix a $\sigma$-derivation $\delta_{s}^{\prime}: A \rtimes \Gamma \rightarrow A \rtimes \Gamma$ for some $s \in \mathbb{N}$.

Proposition 3.8. 11] A connection $\nabla: E \rightarrow E$ for $A$ is a twisted connection for $A \rtimes \Gamma$ if and only if

$$
\nabla \rho(\gamma)=\rho(\gamma)\left((s-1) \pi\left(\delta\left(j \gamma^{-1} \cdot \gamma\right)\right)+\nabla \pi\left(j \gamma^{-1} \cdot \gamma\right)\right), \quad \forall \gamma \in \Gamma .
$$


Remark 3.9. Let $s>0$ be a real number and assume $(j(\gamma))^{s} \in A$ is defined for all $\gamma \in \Gamma$. Propositions 3.5 and 3.8 continue to hold for these values of $s$ as well. For this we need the extra condition $\delta\left(x^{s}\right)=s x^{s-1} \delta(x)$ to hold for all $x=j(\gamma), \gamma \in \Gamma$.

Example 3.10. Let $C^{\infty}\left(S^{1}\right)$ be the algebra of smooth functions on the circle $S^{1}=\mathbb{R} / \mathbb{Z}$, and $\Gamma \subset \operatorname{Diff}^{+}\left(S^{1}\right)$ a group of orientation preserving diffeomorphisms of the circle as in 8 . We represent the algebra $C^{\infty}\left(S^{1}\right)$ by bounded operators in the Hilbert space $L^{2}\left(S^{1}\right)$ by

$$
(\pi(g) \xi)(x)=g(x) \xi(x), \quad \forall g \in C^{\infty}\left(S^{1}\right), \quad \xi \in L^{2}\left(S^{1}\right), \quad x \in \mathbb{R} / \mathbb{Z} .
$$

Define a representation of $\Gamma$ by bounded operators in $L^{2}\left(S^{1}\right)$ by

$$
\left(\rho\left(\phi^{-1}\right) \xi\right)(x)=\phi^{\prime}(x)^{\frac{1}{2}} \xi(\phi(x)), \quad \forall \phi \in \Gamma, \quad \xi \in L^{2}\left(S^{1}\right), \quad x \in \mathbb{R} / \mathbb{Z} .
$$

The group $\Gamma$ acts on $C^{\infty}\left(S^{1}\right)$ from right by composition and one can easily check that the above representations give a covariant system which yields the representation of $C^{\infty}\left(S^{1}\right) \rtimes \Gamma$ as in [8]. The map $j: \Gamma \rightarrow C^{\infty}\left(S^{1}\right)$ defined by $j(\phi)=\phi^{\prime}$ is a 1-cocycle and the derivation $\delta: C^{\infty}\left(S^{1}\right) \rightarrow C^{\infty}\left(S^{1}\right), \delta(f)=\frac{1}{i} f^{\prime}$ is compatible with $j$. Now by using Proposition 3.5. we obtain an automorphism $\sigma$ of $C^{\infty}\left(S^{1}\right) \rtimes \Gamma$ which agrees with the automorphism in [8], and a twisted derivation $\delta_{\frac{1}{2}}^{\prime}$. Note that since it is possible to take the square root of the elements in the image of $j$ in this example, we can let $s=\frac{1}{2}$ to obtain a twisted derivation. Now if we let $\nabla=\frac{1}{i} \frac{d}{d x}$, one can see that the equality (3.2) holds, therefore $\nabla$ is a twisted connection for $C^{\infty}\left(S^{1}\right) \rtimes \Gamma$ by Proposition 3.8.

Also the linear map $\tau: C^{\infty}\left(S^{1}\right) \rightarrow \mathbb{C}$ defined by

$$
\tau(g)=\int_{\mathbb{R} / \mathbb{Z}} g(x) d x, \forall g \in C^{\infty}\left(S^{1}\right),
$$

is a trace which has the change of variable property with respect to $j$, and $\tau \circ \delta=$ 0 . Therefore by Proposition 3.5, one obtains a twisted trace $\tau^{\prime}: C^{\infty}\left(S^{1}\right) \rtimes \Gamma \rightarrow \mathbb{C}$ such that $\tau^{\prime} \circ \delta_{\frac{1}{2}}^{\prime}=0$.

\subsection{Twisting spectral triples by scaling automorphisms}

The last example of the preceding subsection gives a special case of a class of twisted spectral triples that arise naturally in conformal geometry [17. Let $(M, g)$ be a connected compact Riemannian spin manifold of dimension $n$ and $D=D_{g}$ be the associated Dirac operator acting on the Hilbert space of $L^{2}$ spinors $\mathcal{H}=L^{2}\left(M, S^{g}\right)$. Let $S C O(M,[g])$ denote the Lie group of diffeomorphisms of $M$ that preserve the conformal structure $[g]$ consisting of all Riemannian metrics that are conformally equivalent to $g$, the orientation, and the spin structure, and let $G=S C O(M,[g])_{0}$ denote the connected component of the identity. In [17, using a suitable automorphism of the crossed product algebra $C^{\infty}(M) \rtimes G$, a twisted spectral triple of the form $\left(C^{\infty}(M) \rtimes G, L^{2}\left(M, S^{g}\right), D\right)$ is 
constructed. Similarly, by endowing $\mathbb{R}^{n}$ with the Euclidean metric, and considering the group $G$ of conformal transformations of $\mathbb{R}^{n}$, a twisted spectral triple is constructed over the crossed product algebra $C_{c}^{\infty}\left(\mathbb{R}^{n}\right) \rtimes G$. An abstract formulation of this class of twisted spectral triples leads in [17 to the idea of twisting an ordinary spectral triple by its scaling automorphisms or conformal similarities which we explain in this subsection.

Using scaling automorphisms of a spectral triple $(\mathcal{A}, \mathcal{H}, D)$, one can construct a twisted spectral triple [17. The set of scaling automorphisms, also called conformal similarities, of a spectral triple $(\mathcal{A}, \mathcal{H}, D)$, denoted by $\operatorname{Sim}(\mathcal{A}, \mathcal{H}, D)$, consists of all unitary operators $U$ on $\mathcal{H}$ such that

$$
U \mathcal{A} U^{*}=\mathcal{A}, \text { and } U D U^{*}=\mu(U) D, \text { for some } \mu(U)>0 .
$$

It is easy to see that $\operatorname{Sim}(\mathcal{A}, \mathcal{H}, D)$ is a group and the map $\mu: \operatorname{Sim}(\mathcal{A}, \mathcal{H}, D) \rightarrow$ $(0, \infty)$ is a character. We fix a $\operatorname{subgroup} G \subset \operatorname{Sim}(\mathcal{A}, \mathcal{H}, D)$ and let $\mathcal{A}_{G}$ be the crossed product algebra $\mathcal{A} \rtimes G$. It is shown in [17] that the formula

$$
\sigma(a U)=\mu(U)^{-1} a U, \forall a \in \mathcal{A}, U \in G,
$$

defines an automorphism of $\mathcal{A}_{G}$, and $\left(\mathcal{A}_{G}, \mathcal{H}, D\right)$ is a twisted spectral triple. In fact the twisted commutators

$$
[D, a U]_{\sigma}:=D a U-\sigma(a U) D=[D, a] U
$$

are bounded operators for all $a \in \mathcal{A}, U \in G$.

For this class of twisted spectral triples, one can form the crossed product algebra $\Psi(\mathcal{A} \rtimes G, \mathcal{H}, D):=\Psi(\mathcal{A}, \mathcal{H}, D) \rtimes G$, where $\Psi(\mathcal{A}, \mathcal{H}, D)$ is the algebra of pseudodifferential operators associated to the base spectral triple [7, 13, 14, and under the extended simple dimension spectrum hypothesis, the residue functional $f_{D} \Psi\left(\mathcal{A}_{G}, \mathcal{H}, D\right) \rightarrow \mathbb{C}$ given by

$$
f_{D} P:=\operatorname{Res}_{z=0} \operatorname{Trace}\left(P|D|^{-2 z}\right)
$$

is a trace [17. This seems to be in agreement with the twisted analogue of the Adler-Manin trace [1, 16, 11]: For a triple $(A, \sigma, \delta)$ consisting of an algebra $A$, an algebra automorphism $\sigma: A \rightarrow A$, and a $\sigma$-derivation $\delta: A \rightarrow A$, we define an algebra of formal twisted pseudodifferential symbols $\Psi(A, \sigma, \delta)$ whose elements are formal series of the form

$$
\sum_{n=-\infty}^{N} a_{n} \xi^{n}, \quad N \in \mathbb{Z}, a_{n} \in A, \forall n \leq N .
$$

The multiplication in this algebra is essentially derived from these relations

$$
\xi a=\sigma(a) \xi+\delta(a), \forall a \in A, \quad \xi \xi^{-1}=\xi^{-1} \xi=1 .
$$

In [11, we prove that starting from a $\delta$-invariant twisted trace on $A$, the noncommutative residue is a trace on $\Psi(A, \sigma, \delta)$ : 
Theorem 3.11. 11] Let $\sigma: A \rightarrow A$ be an automorphism of an algebra $A$. If $\tau: A \rightarrow \mathbb{C}$ is a $\sigma$-trace and $\delta: A \rightarrow A$ is a $\sigma$-derivation such that $\tau \circ \delta=0$, then the linear functional Res: $\Psi(A, \sigma, \delta) \rightarrow \mathbb{C}$ defined by

$$
\operatorname{Res}\left(\sum_{i=-\infty}^{n} a_{i} \xi^{i}\right)=\tau\left(a_{-1}\right)
$$

is a trace on $\Psi(A, \sigma, \delta)$.

\section{Properties of twisted spectral triples}

In this section, we recall the basic properties of twisted spectral triples [8].

\subsection{Twisted trace}

Given a $\sigma$-spectral triple $(\mathcal{A}, \mathcal{H}, D)$ with $D^{-1} \in \mathcal{L}^{n, \infty}$, it is observed in 8 that

$$
D^{-n} a-\sigma^{-n}(a) D^{-n} \in \mathcal{L}_{0}^{1, \infty}(\mathcal{H}), \quad \forall a \in \mathcal{A} .
$$

Here $\mathcal{L}_{0}^{1, \infty}(\mathcal{H})$ is the ideal

$$
\left\{T \in \mathcal{K}(\mathcal{H}) ; \quad \sum_{i=0}^{N} \mu_{i}(T)=o(\log N)\right\},
$$

on which the Dixmier trace $\operatorname{Tr}_{\omega}$ vanishes. Then it follows that the Dixmier trace induces a twisted hypertrace on $\mathcal{A}$ :

Proposition 4.1. [8] Let $(\mathcal{A}, \mathcal{H}, D)$ be a $\sigma$-spectral triple with $D^{-1} \in \mathcal{L}^{n, \infty}$. Then the linear functional $\varphi: \mathcal{A} \rightarrow \mathbb{C}$ defined by

$$
\varphi(a)=\operatorname{Tr}_{\omega}\left(a D^{-n}\right), \quad a \in \mathcal{A}
$$

is a $\sigma^{n}$-hypertrace, i.e.

$$
\operatorname{Tr}_{\omega}\left(T a D^{-n}\right)=\operatorname{Tr}_{\omega}\left(\sigma^{n}(a) T D^{-n}\right)
$$

for any $a \in \mathcal{A}, T \in \mathcal{L}(\mathcal{H})$. In particular $\varphi$ is a twisted trace on $\mathcal{A}$. Note that for Lipschitz-regular twisted spectral triples, the same holds when $D^{-n}$ is replaced by $|D|^{-n}$.

\subsection{Connes-Chern character}

Let $(\mathcal{A}, \mathcal{H}, D)$ be a Lipschitz-regular $\sigma$-spectral triple such that $D^{-1} \in \mathcal{L}^{n, \infty}(\mathcal{H})$ for some $n \in \mathbb{N}$. Here

$$
\mathcal{L}^{n, \infty}(\mathcal{H})=\left\{T \in \mathcal{K}(\mathcal{H}) ; \quad \sum_{i=0}^{N} \mu_{i}(T)=O\left(N^{1-\frac{1}{n}}\right)\right\}, \text { if } n>1,
$$




$$
\mathcal{L}^{1, \infty}(\mathcal{H})=\left\{T \in \mathcal{K}(\mathcal{H}) ; \quad \sum_{i=0}^{N} \mu_{i}(T)=O(\log N)\right\},
$$

where for any compact operator $T \in \mathcal{K}(\mathcal{H})$, its singular values are written in decreasing order: $\mu_{1}(T) \geq \mu_{2}(T) \geq \cdots \geq 0$.

We recall from [8] that passage to the phase $F=D|D|^{-1}$ of such a twisted spectral triple gives a finitely summable Fredholm module which has a welldefined Connes-Chern character in cyclic cohomology given by

$$
\Phi_{F}\left(a_{0}, a_{1}, \ldots, a_{n}\right)=\operatorname{Trace}\left(\gamma F\left[F, a_{0}\right]\left[F, a_{1}\right] \cdots\left[F, a_{n}\right]\right), a_{0}, a_{1}, \ldots, a_{n} \in \mathcal{A},
$$

where $\gamma=i d$ in the ungraded case. Since for any $a \in \mathcal{A}$

$$
[F, a]=|D|^{-1}\left([D, a]_{\sigma}-[|D|, a]_{\sigma} F\right),
$$

the commutators $\left[F, a_{i}\right]$ are differentials of the same order as $D^{-1}$ and the operator

$$
\gamma F\left[F, a_{0}\right]\left[F, a_{1}\right] \cdots\left[F, a_{n}\right]
$$

is a trace class operator. One can see that $\Phi_{F}$ is a cyclic cocycle, i.e.

$$
\begin{gathered}
\Phi_{F}\left(a_{n}, a_{0}, \ldots, a_{n-1}\right)=(-1)^{n} \Phi_{F}\left(a_{0}, a_{1}, \ldots, a_{n}\right), \forall a_{0}, a_{1}, \ldots, a_{n} \in \mathcal{A}, \\
b \Phi_{F}=0,
\end{gathered}
$$

where $b$ is the Hochschild coboundary operator:

$$
\begin{aligned}
b \Phi_{F}\left(a_{0}, a_{1}, \ldots, a_{n+1}\right)= & \sum_{i=0}^{n}(-1)^{i} \Phi_{F}\left(a_{0}, \ldots, a_{i} a_{i+1}, \ldots, a_{n+1}\right)+ \\
& (-1)^{n+1} \Phi_{F}\left(a_{n+1} a_{0}, a_{1}, \ldots, a_{n}\right),
\end{aligned}
$$

for all $a_{0}, a_{1}, \ldots, a_{n+1} \in \mathcal{A}$.

Moreover, if $D^{-1} \in \mathcal{L}^{n, \infty}(\mathcal{H})$ for an even $n \in \mathbb{N}$, then the Connes-Chern character can be defined without the Lipschitz-regularity assumption:

Proposition 4.2. 8] Let $(\mathcal{A}, \mathcal{H}, D)$ be a graded $\sigma$-spectral triple with $D^{-1} \in$ $\mathcal{L}^{n, \infty}(\mathcal{H})$ for some even $n \in \mathbb{N}$. Then the multilinear functional $\Phi_{D, \sigma}$ defined by

$$
\Phi_{D, \sigma}\left(a_{0}, a_{1}, \ldots, a_{n}\right)=\operatorname{Trace}\left(\gamma D^{-1}\left[D, a_{0}\right]_{\sigma} D^{-1}\left[D, a_{1}\right]_{\sigma} \cdots D^{-1}\left[D, a_{n}\right]_{\sigma}\right)
$$

for $a_{0}, \ldots, a_{n} \in \mathcal{A}$, is a cyclic cocycle.

\subsection{Index pairing and $K$-theory}

In [8], it is shown that in the twisted case, the index pairing with ordinary $K$ theory makes sense and it is given by the pairing of the Connes-Chern character with $K$-theory. 
Given a graded $\sigma$-spectral triple $(\mathcal{A}, \mathcal{H}, D)$ with $D^{-1} \in \mathcal{L}^{n, \infty}(\mathcal{H})$ for some even $n \in \mathbb{N}$, one can write an orthogonal decomposition for the Hilbert space $\mathcal{H}$ using the grading $\gamma$ :

$$
\mathcal{H}=\mathcal{H}_{+} \oplus \mathcal{H}_{-}
$$

where

$$
\mathcal{H}_{+}=\{x \in \mathcal{H} ; \quad \gamma(x)=x\}, \mathcal{H}_{-}=\{x \in \mathcal{H} ; \quad \gamma(x)=-x\} .
$$

Now a close look at the Connes-Chern character $\Phi_{D, \sigma}$ defined by (4.3) shows the existence of a pair of Fredholm modules over $\mathcal{A}$ and two cocycles as follows. With respect to the decomposition $\mathcal{H}=\mathcal{H}_{+} \oplus \mathcal{H}_{-}$, we can write

$$
D=\left[\begin{array}{cc}
0 & D_{-} \\
D_{+} & 0
\end{array}\right], \quad a=\left[\begin{array}{cc}
a_{+} & 0 \\
0 & a_{-}
\end{array}\right], \forall a \in \mathcal{A}
$$

since we have $D \gamma=-\gamma D$, and $a \gamma=\gamma a$, for all $a \in \mathcal{A}$. Then define two Hilbert spaces

$$
\widetilde{\mathcal{H}}^{+}=\mathcal{H}_{+} \oplus \mathcal{H}_{+}, \quad \widetilde{\mathcal{H}}^{-}=\mathcal{H}_{-} \oplus \mathcal{H}_{-} .
$$

There are representations $\pi^{+}$and $\pi^{-}$of the algebra $\mathcal{A}$ on $\mathcal{L}\left(\widetilde{\mathcal{H}}^{+}\right)$and $\mathcal{L}\left(\widetilde{\mathcal{H}}^{-}\right)$ respectively given by

$$
\pi^{ \pm}(a)=\left[\begin{array}{cc}
a_{ \pm} & 0 \\
0 & D_{ \pm}^{-1} \sigma(a)_{\mp} D_{ \pm}
\end{array}\right]
$$

In $[8$, letting

$$
F=\left[\begin{array}{cc}
0 & I_{ \pm} \\
I_{ \pm} & 0
\end{array}\right]
$$

where $I_{+}$and $I_{-}$are identity operators on $\mathcal{H}_{+}$and $\mathcal{H}_{-}$respectively, it is shown that the commutators $\left[F^{ \pm}, \pi^{ \pm}(a)\right]$ are compact operators, hence they obtain a pair of Fredholm modules over the algebra $\mathcal{A}$. It is also shown that for any idempotent $e \in \mathcal{A}$, the bounded closure of operators $D_{ \pm}^{-1} \sigma(e)_{\mp} D_{ \pm}$denoted by $f_{ \pm}$are idempotents, and $f_{ \pm} e_{ \pm}: e_{ \pm} \mathcal{H}_{ \pm} \rightarrow f_{ \pm} \mathcal{H}_{ \pm}$are Fredholm operators. Since the index depends only on the $K$-theory class of the idempotent, they define a pair of index maps by

$$
\operatorname{Index}^{ \pm}[e]=\operatorname{Index}\left(f_{ \pm} e_{ \pm}\right),
$$

for all idempotents $e \in M_{N}(\mathcal{A})$.

On the other hand, the cyclic cocycle $\Phi_{D, \sigma}$ defined by (4.3) consists of a pair of cocycles $\Phi_{D, \sigma}^{ \pm}$given by

$$
\begin{aligned}
& \Phi_{D, \sigma}^{ \pm}\left(a^{0}, \ldots, a^{n}\right) \\
= & \operatorname{Trace}\left(D_{ \pm}^{-1}\left(D_{ \pm} a_{ \pm}^{0}-\sigma\left(a^{0}\right)_{\mp} D_{ \pm}\right) \cdots D_{ \pm}^{-1}\left(D_{ \pm} a_{ \pm}^{n}-\sigma\left(a^{n}\right)_{\mp} D_{ \pm}\right)\right),
\end{aligned}
$$

for $a^{0}, \ldots, a^{n} \in \mathcal{A}$.

The following proposition states that the index pairing can be expressed as the pairing of these cocycles with $K$-theory: 
Proposition 4.3. 8] Given a graded $\sigma$-spectral triple $(\mathcal{A}, \mathcal{H}, D)$ with $D^{-1} \in$ $\mathcal{L}^{n, \infty}(\mathcal{H})$ for some even $n \in \mathbb{N}$, and an idempotent $e \in M_{N}(\mathcal{A})$, one has

$$
\operatorname{Index}^{ \pm}[e]=\Phi_{D, \sigma}^{ \pm}(e, \ldots, e) \text {. }
$$

If $e^{*}=\sigma(e)$, then

$$
\operatorname{Index}^{+}[e]=-\operatorname{Index}^{-}[e] \text {. }
$$

If there exists a strongly continuous 1-parameter group of isometric automorphisms $\left\{\sigma_{t}\right\}_{t \in \mathbb{R}}$ with an analytic extension coinciding with $\sigma$ at $t=-i$, then the above index maps coincide. This assumption is denoted by (1PG). Such an analytic extension defined on a dense subalgebra $\mathcal{O}$ of $\mathcal{A}$ is assured to exist by a theorem of Bost in [2].

Proposition 4.4. 8] If $\mathcal{A}$ satisfies (1PG), then

$$
\operatorname{Index}^{+}[e]=-\operatorname{Index}^{-}[e], \forall e \in M_{N}(\mathcal{A}) \text {. }
$$

Accordingly, in 8 , the relation between the cyclic cocycles $\Phi_{D, \sigma}^{ \pm}$has been studied under the (1PG) assumption.

Theorem 4.5. 8] Let $(\mathcal{A}, \mathcal{H}, D)$ be a graded $\sigma$-spectral triple as above and assume that $\mathcal{A}$ satisfies $(1 P G)$. Then

$$
\left[\Phi_{D, \sigma}^{-}\right]=-\left[\left(\Phi_{D, \sigma}^{+}\right)^{*}\right] \in H P^{e v}(\mathcal{O})
$$

where

$$
\left(\Phi_{D, \sigma}^{+}\right)^{*}\left(a_{0}, \ldots, a_{n}\right):=\overline{\Phi_{D, \sigma}^{+}\left(a_{n}^{*}, \ldots, a_{0}^{*}\right)}, \forall a_{0}, \ldots, a_{n} \in \mathcal{O} .
$$

We note that in the proof of the latter 8 , the homotopy invariance of the Connes-Chern character of a finitely summable Fredholm module, established in Lemma 1, in Part I, section 5 of [3] plays a crucial role.

\subsection{Local Hochschild cocycle}

In [8, as a first step to extend the local index formula [7, 13, 14, to twisted spectral triples, using the Dixmier trace, a local Hochschild cocycle is constructed for twisted spectral triples:

Proposition 4.6. 8] Let $(\mathcal{A}, \mathcal{H}, D)$ be a graded $\sigma$-spectral triple such that $D^{-1} \in \mathcal{L}^{n, \infty}(\mathcal{H})$ for some even $n \in \mathbb{N}$. Then the $n+1$-linear form on $\mathcal{A}$ defined by

$$
\begin{aligned}
& \Psi_{D, \sigma}\left(a_{0}, a_{1}, \ldots, a_{n}\right) \\
= & \operatorname{Tr}_{\omega}\left(\gamma a_{0}\left[D, \sigma^{-1}\left(a_{1}\right)\right]_{\sigma} \cdots\left[D, \sigma^{-n}\left(a_{n}\right)\right]_{\sigma}|D|^{-n}\right)
\end{aligned}
$$

for $a_{0}, \ldots, a_{n} \in \mathcal{A}$, is a Hochschild cocycle.

In the ungraded case, for a Lipschitz-regular $\sigma$-spectral triple of odd summability degree, the Hochschild cocycle is given by

$$
\begin{aligned}
& \Psi_{D, \sigma}\left(a_{0}, a_{1}, \ldots, a_{n}\right) \\
= & \operatorname{Tr}_{\omega}\left(a_{0}\left[D, \sigma^{-1}\left(a_{1}\right)\right]_{\sigma} \cdots\left[D, \sigma^{-n}\left(a_{n}\right)\right]_{\sigma}|D|^{-n}\right),
\end{aligned}
$$

for any $a_{0}, \ldots, a_{n} \in \mathcal{A}$. 
The above cocycle identities are proved in 8$]$ using Proposition 4.1 and the fact that for any $a, b \in \mathcal{A}$ :

$$
[D, a b]_{\sigma}=[D, a]_{\sigma} b+\sigma(a)[D, b]_{\sigma} .
$$

In [8, 17, the above local Hochschild cocycle is obtained in a heuristic manner as follows. For an ordinary spectral triple $(\mathcal{A}, \mathcal{H}, D)$, consider the local Hochschild cocycle

$$
\Psi_{D}\left(a_{0}, \ldots, a_{n}\right)=\operatorname{Tr}_{\omega}\left(\gamma a_{0}\left[D, a_{1}\right] \cdots\left[D, a_{n}\right] D^{-n}\right),
$$

for any $a_{0}, \ldots, a_{n} \in \mathcal{A}$. One can move $D^{-n}$ to the left and distribute it among the factors to write this cocycle in the form:

$\Psi_{D}\left(a_{0}, \ldots, a_{n}\right)=\operatorname{Tr}_{\omega}\left(\gamma a_{0}\left(D a_{1} D^{-1}-a_{1}\right) \cdots\left(D^{n} a_{n} D^{-n}-D^{n-1} a_{n} D^{-n+1}\right) D^{-n}\right)$.

In order to construct a local Hochschild cocycle for twisted spectral triples, they replace each $D^{k} a D^{-k}$ in the latter by $D^{k} \sigma^{-k}(a) D^{-k}$ and reverse the process of moving the $D^{-n}$ to the left which leads to the above local Hochschild cocycle for twisted spectral triples. We note that the Connes-Chern character introduced in Proposition 4.2 can be obtained in a similar manner.

\section{Hochschild class of the Connes-Chern charac- ter}

For any algebra $\mathcal{A}$, there is an obvious pairing between the space of Hochschild $n$-cochains $C^{n}\left(\mathcal{A}, \mathcal{A}^{*}\right)$, i.e. the space of $(n+1)$-linear functionals on $\mathcal{A}$, and the space of Hochschild $n$-chains $\mathcal{A}^{\otimes(n+1)}$, given by

$$
\left\langle\varphi, a_{0} \otimes a_{1} \otimes \cdots \otimes a_{n}\right\rangle:=\varphi\left(a_{0}, \ldots, a_{n}\right) .
$$

This pairing satisfies

$$
\langle b \varphi, c\rangle=\langle\varphi, b(c)\rangle
$$

where the Hochschild operators for the cochains and chains are given by:

$$
\begin{aligned}
b \varphi\left(a_{0}, a_{1}, \ldots, a_{n+1}\right)= & \sum_{i=0}^{n}(-1)^{i} \varphi\left(a_{0}, \ldots, a_{i} a_{i+1}, \ldots, a_{n+1}\right)+ \\
& (-1)^{n+1} \varphi\left(a_{n+1} a_{0}, a_{1}, \ldots, a_{n}\right), \\
b\left(a_{0} \otimes a_{1} \otimes \cdots \otimes a_{n+1}\right)= & \sum_{i=0}^{n}(-1)^{i} a_{0} \otimes \cdots \otimes a_{i} a_{i+1} \otimes \cdots \otimes a_{n+1}+ \\
& (-1)^{n+1} a_{n+1} a_{0} \otimes a_{1} \otimes \cdots \otimes a_{n},
\end{aligned}
$$


for $a_{0}, \ldots, a_{n+1} \in \mathcal{A}$. It follows that, if two cocycles $\varphi_{1}$ and $\varphi_{2}$ are cohomologous with $\varphi_{1}-\varphi_{2}=b \psi$, then they yield the same value on any Hochschild cycle since we have

$$
\left\langle\varphi_{1}, c\right\rangle-\left\langle\varphi_{2}, c\right\rangle=\langle b \psi, c\rangle=\langle\psi, b(c)\rangle=0 \text { if } b(c)=0 .
$$

As we saw in Section 4 to any twisted spectral triple, in particular to an ordinary spectral triple, with certain conditions one can associate the ConnesChern character and a local Hochschild cocycle. Connes' character formula (or Connes' Hochschild character theorem) states that in the case of ordinary spectral triples, these two cocycles yield the same value on any Hochschild cycle [5, 12, 14.

In this section the analogue of Connes' character formula for the class of twisted spectral triples introduced in Subsection 3.3 is investigated which we explain next following [10].

\subsection{Connes' character formula and twisting by scaling au- tomorphisms}

In Subsection 3.3. we saw that using conformal similarities of a spectral triple $(\mathcal{A}, \mathcal{H}, D)$, one can construct a twisted spectral triple $(c f$. [17]). We recall that the set of conformal similarities of a spectral triple $(\mathcal{A}, \mathcal{H}, D)$, denoted by $\operatorname{Sim}(\mathcal{A}, \mathcal{H}, D)$, consists of all unitary operators $U$ on $\mathcal{H}$, such that

$$
U \mathcal{A} U^{*}=\mathcal{A} \text {, and } U D U^{*}=\mu(U) D, \text { for some } \mu(U)>0 .
$$

Recall that $\operatorname{Sim}(\mathcal{A}, \mathcal{H}, D)$ is a group and the map $\mu: \operatorname{Sim}(\mathcal{A}, \mathcal{H}, D) \rightarrow(0, \infty)$ is a character. We fix a subgroup $G \subset \operatorname{Sim}(\mathcal{A}, \mathcal{H}, D)$ and let $\mathcal{A}_{G}$ be the crossed product algebra $\mathcal{A} \rtimes G$. The formula

$$
\sigma(a U)=\mu(U)^{-1} a U, \quad \forall a \in \mathcal{A}, U \in G,
$$

defines an automorphism of $\mathcal{A}_{G}$, and $\left(\mathcal{A}_{G}, \mathcal{H}, D\right)$ is a $\sigma$-spectral triple [17. We will assume that the base spectral triple $(\mathcal{A}, \mathcal{H}, D)$ is regular, i.e. the operators $\mathcal{A},[D, \mathcal{A}]$ are in the domain of all powers of the derivation $\delta(\cdot)=[|D|, \cdot]$. We also assume that $D^{-1} \in \mathcal{L}^{n, \infty}(\mathcal{H})$ for some fixed even number $n \in 2 \mathbb{N}$, and that the twisted spectral triple $\left(\mathcal{A}_{G}, \mathcal{H}, D\right)$ is graded.

From the regularity of the base spectral triple, it easily follows that the twisted spectral triple $\left(\mathcal{A}_{G}, \mathcal{H}, D\right)$ is Lipschitz-regular. Therefore, by passage to the phase, one can associate the Connes-Chern character to the Fredholm module $\left(\mathcal{A}_{G}, \mathcal{H}, F=D|D|^{-1}\right)$. To recall, this is a cyclic $n$-cocycle given by

$$
\Phi_{F}\left(a_{0} U_{0}, a_{1} U_{1}, \ldots, a_{n} U_{n}\right)=\operatorname{Trace}\left(\gamma F\left[F, a_{0} U_{0}\right]\left[F, a_{1} U_{1}\right] \cdots\left[F, a_{n} U_{n}\right]\right)
$$

for all $a_{i} \in \mathcal{A}, U_{i} \in G, i=0, \ldots, n$. Here $\gamma$ denotes the grading. Also there is a Hochschild $n$-cocycle given by

$$
\begin{aligned}
& \Psi_{D, \sigma}\left(a_{0} U_{0}, a_{1} U_{1}, \ldots, a_{n} U_{n}\right) \\
= & \operatorname{Tr}_{\omega}\left(\gamma a_{0} U_{0}\left[D, \sigma^{-1}\left(a_{1} U_{1}\right)\right]_{\sigma} \cdots\left[D, \sigma^{-n}\left(a_{n} U_{n}\right)\right]_{\sigma}|D|^{-n}\right)
\end{aligned}
$$


for all $a_{i} \in \mathcal{A}, U_{i} \in G, i=0, \ldots, n$.

We recall from 12 how

$$
\Phi_{F}\left(a_{0} U_{0}, a_{1} U_{1}, \ldots, a_{n} U_{n}\right)=\operatorname{Trace}\left(\gamma F\left[F, a_{0} U_{0}\right]\left[F, a_{1} U_{1}\right] \cdots\left[F, a_{n} U_{n}\right]\right)
$$

can be approximated by the trace of finite rank operators using a cutoff. Let $g: \mathbb{R} \rightarrow \mathbb{R}$ be a smooth function which takes the value 1 on $\left[0, \frac{1}{2}\right]$, decreases to 0 on $\left[\frac{1}{2}, 1\right]$, is 0 for $t>1, g(-t)=g(t)$ for $t<0$, and $\int_{0}^{\infty} g^{\prime}(u) \mathrm{d} u=-1$. Define the operators $A_{t}=g(t|D|)$ for $t>0$. The operators $A_{t}, t>0$, are positive, finite rank, and satisfy $P_{1 / 2 t} \leq A_{t} \leq P_{1 / t}$, where $P_{N}$ is the spectral projector of $|D|$ on the interval $[0, N]$. Given a Hochschild $n$-cycle

$$
c=\sum_{j=1}^{k} a_{0 j} U_{0 j} \otimes a_{1 j} U_{1 j} \otimes \cdots \otimes a_{n j} U_{n j},
$$

it is proved in [12] that

$$
\Phi_{F}(c)=2 \lim _{t \downarrow 0} \Psi_{t}(c),
$$

where

$$
\begin{aligned}
& \Psi_{t}\left(a_{0} U_{0}, a_{1} U_{1}, \ldots, a_{n} U_{n}\right) \\
:= & -\operatorname{Trace}\left(\gamma a_{0} U_{0}\left[F, a_{1} U_{1}\right] \cdots\left[F, a_{n-1} U_{n-1}\right] F\left[A_{t}, a_{n} U_{n}\right]\right) .
\end{aligned}
$$

The proof of this is essentially based on two facts ( $c f$. 12). The first is that the operator trace is normal, and the second is that, since $b(c)=0$, one has

$$
\begin{aligned}
& \sum_{j=1}^{k} a_{0 j} U_{0 j}\left[F, a_{1 j} U_{1 j}\right] \cdots\left[F, a_{n-1, j} U_{n-1, j}\right] a_{n j} U_{n j} \\
= & \sum_{j=1}^{k} a_{n j} U_{n j} a_{0 j} U_{0 j}\left[F, a_{1 j} U_{1 j}\right] \cdots\left[F, a_{n-1, j} U_{n-1, j}\right] .
\end{aligned}
$$

For convenience, we drop the index $j$ and the summation in the formula for the Hochschild cycle $c$, and simply write

$$
c=a_{0} U_{0} \otimes a_{1} U_{1} \otimes \cdots \otimes a_{n} U_{n} .
$$

Lemma 5.1. [10] The operator $a_{0} U_{0}\left[F, a_{1} U_{1}\right] \cdots\left[F, a_{n-1} U_{n-1}\right] F|D|^{n-1}$ is bounded.

Proof. Using the identity $U[F, a]=\left[F, U a U^{*}\right] U$, for all $a \in A, U \in G$, one can see that

$$
\begin{aligned}
& a_{0} U_{0}\left[F, a_{1} U_{1}\right] \cdots\left[F, a_{n-1} U_{n-1}\right] F|D|^{n-1} \\
= & \mu\left(U_{0} U_{1} \cdots U_{n-1}\right)^{n-1} a_{0}\left[F, U_{0} a_{1} U_{0}^{*}\right] \cdots \\
& {\left[F, U_{0} U_{1} \cdots U_{n-2} a_{n-1} U_{n-2}^{*} \cdots U_{1}^{*} U_{0}^{*}\right] F|D|^{n-1} U_{0} U_{1} \cdots U_{n-1} . }
\end{aligned}
$$

The boundedness of the latter follows from the fact that the operator

$$
a_{0}^{\prime}\left[F, a_{1}^{\prime}\right] \cdots\left[F, a_{n-1}^{\prime}\right] F|D|^{n-1}
$$

is bounded for any $a_{0}^{\prime}, \ldots, a_{n-1}^{\prime} \in A$, which is proved in [12]. 
For convenience let

$$
R=-\gamma a_{0} U_{0}\left[F, a_{1} U_{1}\right] \cdots\left[F, a_{n-1} U_{n-1}\right] F|D|^{n-1} \in \mathcal{L}(H) .
$$

Then we have:

$$
\Psi_{t}\left(a_{0} U_{0}, a_{1} U_{1}, \ldots, a_{n} U_{n}\right)=\operatorname{Trace}\left(R|D|^{-(n-1)}\left[A_{t}, a_{n} U_{n}\right]\right) .
$$

In the sequel we will impose the following extra condition on the Hochschild cycle $c=a_{0} U_{0} \otimes a_{1} U_{1} \otimes \cdots \otimes a_{n} U_{n}$ (note that we have dropped the summation):

Condition 5.2. We shall assume that

$$
\lim _{t \downarrow 0} \operatorname{Trace}\left(R|D|^{-(n-1)}\left[A_{t}, a_{n} U_{n}\right]\right)=\lim _{t \downarrow 0} \operatorname{Trace}\left(R|D|^{-(n-1)}\left[A_{t}, a_{n}\right] U_{n}\right) .
$$

For example, if $\mu\left(U_{n}\right)=1$ then this condition is satisfied.

The function $\Psi_{t}^{\prime}\left(a_{0} U_{0}, a_{1} U_{1}, \ldots, a_{n} U_{n}\right):=\operatorname{Trace}\left(R|D|^{-(n-1)}\left[A_{t}, a_{n}\right] U_{n}\right)$ is continuous on the interval $\frac{1}{t} \geq e$, and has a limit as $\frac{1}{t} \rightarrow \infty$, therefore it is bounded. Hence the evaluation of the state $\omega$ on the corresponding element in the $C^{*}$-algebra $C_{b}([e, \infty)) / C_{0}([e, \infty))$ will yield this limit which will be denoted by

$$
\lim _{t^{-1} \rightarrow \omega} \Psi_{t}^{\prime}\left(a_{0} U_{0}, a_{1} U_{1}, \ldots, a_{n} U_{n}\right) .
$$

To compute this limit, one can use the following proposition of Connes. For a detailed discussion, we refer the reader to [12].

Proposition 5.3. Let $f:[0, \infty) \rightarrow[0, \infty)$ be a continuous function, $p>1$, and $\sum_{k} m_{k}(f) e^{p k}<\infty$, where for each $k$

$$
m_{k}(f)=\sup \{f(u) ; \quad k \leq \log u \leq k+1\} .
$$

Then $M_{p}=\int_{0}^{\infty} f(u) u^{p-1} \mathrm{~d} u$ is finite and

$$
\lim _{t^{-1} \rightarrow \omega} t^{p} \operatorname{Trace}(f(t|D|) S)=M_{p} \operatorname{Tr}_{\omega}\left(S|D|^{-p}\right),
$$

provided $S \in \mathcal{L}(\mathcal{H})$, and $D^{-1} \in \mathcal{L}^{p, \infty}(\mathcal{H})$.

Also we use Lemma 10.29 of [12].

Lemma 5.4. If $g(t)=h(t)^{2}$ where $h \in \mathcal{D}(\mathbb{R})$ is also a cutoff, and if $a \in \mathcal{A}$, then

$$
\left\|[g(t|D|), a]-\frac{1}{2}\left\{g^{\prime}(t|D|), t \delta a\right\}\right\|_{n-}=o(t) \text { as } t \downarrow 0 .
$$

We note that $\|\cdot\|_{n-}$ is defined in [12].

The argument following the above lemma in [12] applies verbatim to our case by simply replacing $R$ by $U_{n} R$, and it follows that

$$
\begin{aligned}
& \lim _{t^{-1} \rightarrow \omega} \Psi_{t}^{\prime}\left(a_{0} U_{0}, a_{1} U_{1}, \ldots, a_{n} U_{n}\right) \\
= & n \operatorname{Tr}_{\omega}\left(\gamma a_{0} U_{0}\left[F, a_{1} U_{1}\right] \cdots\left[F, a_{n-1} U_{n-1}\right] D^{-1} \delta\left(a_{n}\right) U_{n}\right) \\
= & n \operatorname{Tr}_{\omega}\left(\gamma a_{0} U_{0}\left[F, a_{1} U_{1}\right] \cdots\left[F, a_{n-1} U_{n-1}\right] \delta_{\sigma} \sigma^{-1}\left(a_{n} U_{n}\right) D^{-1}\right),
\end{aligned}
$$


where $\delta(x)=[|D|, x]$ and $\delta_{\sigma}(x)=[|D|, x]_{\sigma}$.

The last step is to prove that the local Hochschild cocycle $\Psi_{D, \sigma}$ is cohomologous to $\zeta_{n}^{\sigma}$ defined by

$$
\begin{aligned}
& \zeta_{n}^{\sigma}\left(a_{0} U_{0}, a_{1} U_{1}, \ldots, a_{n} U_{n}\right) \\
= & n \operatorname{Tr}_{\omega}\left(\gamma a_{0} U_{0}\left[F, a_{1} U_{1}\right] \cdots\left[F, a_{n-1} U_{n-1}\right] \delta_{\sigma} \sigma^{-1}\left(a_{n} U_{n}\right) D^{-1}\right)
\end{aligned}
$$

for any $a_{i} \in \mathcal{A}$, and $U_{i} \in G, i=0, \ldots, n$. This will be achieved by defining the cochains $\zeta_{1}^{\sigma}, \ldots, \zeta_{n-1}^{\sigma}$ by defining $\zeta_{k}^{\sigma}\left(a_{0} U_{0}, a_{1} U_{1}, \ldots, a_{n} U_{n}\right)$ as

$$
n \operatorname{Tr}_{\omega}\left(\gamma a_{0} U_{0}\left[F, a_{1} U_{1}\right] \cdots\left[F, a_{k-1} U_{k-1}\right] D^{-1} \delta_{\sigma}\left(a_{k} U_{k}\right)\left[F, a_{k+1} U_{k+1}\right] \cdots\left[F, a_{n} U_{n}\right]\right)
$$

for any $a_{i} \in \mathcal{A}, U_{i} \in G, i=0, \ldots, n$. Then using the following two lemmas, one can see that $\Psi_{D, \sigma}$ is cohomologous to $\zeta_{n}^{\sigma}$, hence they yield the same value on any Hochschild cycle.

Lemma 5.5. 10 The cochains $\zeta_{1}^{\sigma}, \ldots, \zeta_{n}^{\sigma}$ are mutually cohomologous Hochschild cocycles.

Proof. First we show that $b \zeta_{n}^{\sigma}=0$. For any $a_{i} \in \mathcal{A}, U_{i} \in G$, we have

$$
\begin{aligned}
& b \zeta_{n}^{\sigma}\left(a_{0} U_{0}, \ldots, a_{n+1} U_{n+1}\right) \\
= & \operatorname{Tr}_{\omega}\left(\gamma a_{0} U_{0} a_{1} U_{1}\left[F, a_{2} U_{2}\right] \cdots\left[F, a_{n} U_{n}\right]\left[|D|, \sigma^{-1}\left(a_{n+1} U_{n+1}\right)\right]_{\sigma} D^{-1}\right)- \\
& \operatorname{Tr}_{\omega}\left(\gamma a_{0} U_{0}\left[F, a_{1} U_{1} a_{2} U_{2}\right] \cdots\left[F, a_{n} U_{n}\right]\left[|D|, \sigma^{-1}\left(a_{n+1} U_{n+1}\right)\right]_{\sigma} D^{-1}\right)- \\
& \cdots+ \\
& \operatorname{Tr}_{\omega}\left(\gamma a_{0} U_{0}\left[F, a_{1} U_{1}\right] \cdots\left[F, a_{n-1} U_{n-1}\right]\left[|D|, \sigma^{-1}\left(a_{n} U_{n} a_{n+1} U_{n+1}\right)\right]_{\sigma} D^{-1}\right) \\
& -\operatorname{Tr}_{\omega}\left(\gamma a_{n+1} U_{n+1} a_{0} U_{0}\left[F, a_{1} U_{1}\right] \cdots\left[F, a_{n-1} U_{n-1}\right]\left[|D|, \sigma^{-1}\left(a_{n} U_{n}\right)\right]_{\sigma} D^{-1}\right) \\
= & \operatorname{Tr}_{\omega}\left(\gamma a_{0} U_{0} a_{1} U_{1}\left[F, a_{2} U_{2}\right] \cdots\left[F, a_{n} U_{n}\right]\left[|D|, \sigma^{-1}\left(a_{n+1} U_{n+1}\right)\right]_{\sigma} D^{-1}\right) \\
& -\operatorname{Tr}_{\omega}\left(\gamma a_{0} U_{0} a_{1} U_{1}\left[F, a_{2} U_{2}\right] \cdots\left[F, a_{n} U_{n}\right]\left[|D|, \sigma^{-1}\left(a_{n+1} U_{n+1}\right)\right]_{\sigma} D^{-1}\right) \\
& -\operatorname{Tr}_{\omega}\left(\gamma a_{0} U_{0}\left[F, a_{1} U_{1}\right] a_{2} U_{2} \cdots\left[F, a_{n} U_{n}\right]\left[|D|, \sigma^{-1}\left(a_{n+1} U_{n+1}\right)\right]_{\sigma} D^{-1}\right) \\
& \cdots \\
& +\operatorname{Tr}_{\omega}\left(\gamma a_{0} U_{0}\left[F, a_{1} U_{1}\right] \cdots\left[F, a_{n-1} U_{n-1}\right] a_{n} U_{n}\left[|D|, \sigma^{-1}\left(a_{n+1} U_{n+1}\right)\right]_{\sigma} D^{-1}\right) \\
& +\operatorname{Tr}_{\omega}\left(\gamma a_{0} U_{0}\left[F, a_{1} U_{1}\right] \cdots\left[F, a_{n-1} U_{n-1}\right]\left[|D|, \sigma^{-1}\left(a_{n} U_{n}\right)\right]_{\sigma}\right. \\
& \left.\quad \sigma^{-1}\left(a_{n+1} U_{n+1}\right) D^{-1}\right) \\
& -\operatorname{Tr}_{\omega}\left(\gamma a_{n+1} U_{n+1} a_{0} U_{0}\left[F, a_{1} U_{1}\right] \cdots\left[F, a_{n-1} U_{n-1}\right]\left[|D|, \sigma^{-1}\left(a_{n} U_{n}\right)\right]_{\sigma} D^{-1}\right) .
\end{aligned}
$$

The latter vanishes because of successive cancellations, where the last two terms cancel each other since using the identity

$$
D^{-1} a_{n+1} U_{n+1}-\sigma^{-1}\left(a_{n+1} U_{n+1}\right) D^{-1}=-D^{-1}\left[D, \sigma^{-1}\left(a_{n+1} U_{n+1}\right)\right]_{\sigma} D^{-1},
$$

one obtains a trace class operator which vanishes under the Dixmier trace.

Now we introduce cochains $\eta_{1}^{\sigma}, \ldots, \eta_{n-1}^{\sigma}$ such that $\zeta_{k}^{\sigma}-\zeta_{k+1}^{\sigma}=b \eta_{k}^{\sigma}$ for all $k=1, \ldots, n-1$, and this will finish the proof. 
Using the identities

$$
\begin{gathered}
D^{-1} \delta_{\sigma}\left(a_{k} U_{k}\right)-\delta_{\sigma} \sigma^{-1}\left(a_{k} U_{k}\right) D^{-1}=-D^{-1} \delta\left(\left[D, a_{k}\right]\right) D^{-1} U_{k}, \\
|D|^{-1}\left[F, a_{j} U_{j}\right]-\left[F, \sigma^{-1}\left(a_{j} U_{j}\right)\right]|D|^{-1}=-|D|^{-1}\left[F, \delta_{\sigma} \sigma^{-1}\left(a_{j} U_{j}\right)\right]|D|^{-1}, \\
F\left[F, a_{j} U_{j}\right]=-\left[F, a_{j} U_{j}\right] F,
\end{gathered}
$$

we can move $D^{-1}$ in the formula for $\zeta_{k}^{\sigma}$ to the right under the Dixmier trace and obtain the following expression.

$$
\begin{aligned}
& \zeta_{k}^{\sigma}\left(a_{0} U_{0}, a_{1} U_{1}, \ldots, a_{n} U_{n}\right) \\
= & n \operatorname{Tr}_{\omega}\left(\gamma a_{0} U_{0}\left[F, a_{1} U_{1}\right] \cdots\left[F, a_{k-1} U_{k-1}\right]\right. \\
& \left.D^{-1} \delta_{\sigma}\left(a_{k} U_{k}\right)\left[F, a_{k+1} U_{k+1}\right] \cdots\left[F, a_{n} U_{n}\right]\right) \\
= & (-1)^{n-k} n \operatorname{Tr}_{\omega}\left(\gamma a_{0} U_{0}\left[F, a_{1} U_{1}\right] \cdots\left[F, a_{k-1} U_{k-1}\right]\right. \\
& \left.\delta_{\sigma}\left(\sigma^{-1}\left(a_{k} U_{k}\right)\right)\left[F, \sigma^{-1}\left(a_{k+1} U_{k+1}\right)\right] \cdots\left[F, \sigma^{-1}\left(a_{n} U_{n}\right)\right] D^{-1}\right) .
\end{aligned}
$$

Therefore $\left(\zeta_{k}^{\sigma}-\zeta_{k+1}^{\sigma}\right)\left(a_{0} U_{0}, a_{1} U_{1}, \ldots, a_{n} U_{n}\right)$ is equal to

$$
\begin{array}{r}
(-1)^{n-k} n \operatorname{Tr}_{\omega}\left(\gamma a_{0} U_{0}\left[F, a_{1} U_{1}\right] \cdots\left[F, a_{k-1} U_{k-1}\right] R_{k}^{\sigma}\left[F, \sigma^{-1}\left(a_{k+2} U_{k+2}\right)\right]\right. \\
\left.\cdots\left[F, \sigma^{-1}\left(a_{n} U_{n}\right)\right] D^{-1}\right),
\end{array}
$$

where

$$
R_{k}^{\sigma}=\delta_{\sigma} \sigma^{-1}\left(a_{k} U_{k}\right)\left[F, \sigma^{-1}\left(a_{k+1} U_{k+1}\right)\right]+\left[F, a_{k} U_{k}\right] \delta_{\sigma} \sigma^{-1}\left(a_{k+1} U_{k+1}\right) .
$$

Now we define

$$
\begin{aligned}
& \eta_{k}^{\sigma}\left(a_{0} U_{0}, \ldots, a_{n-1} U_{n-1}\right) \\
= & (-1)^{k} n \operatorname{Tr}_{\omega}\left(\gamma a_{0} U_{0}\left[F, a_{1} U_{1}\right] \cdots\left[F, a_{k-1} U_{k-1}\right]\right. \\
& {\left.\left[F, \delta_{\sigma} \sigma^{-1}\left(a_{k} U_{k}\right)\right]\left[F, \sigma^{-1}\left(a_{k+1} U_{k+1}\right)\right] \cdots\left[F, \sigma^{-1}\left(a_{n-1} U_{n-1}\right)\right] D^{-1}\right) . }
\end{aligned}
$$

Finally, using the identity

$$
\begin{aligned}
& {\left[F, \delta_{\sigma} \sigma^{-1}\left(a_{k} U_{k} a_{k+1} U_{k+1}\right)\right] } \\
= & R_{k}^{\sigma}+\left[F, \delta_{\sigma} \sigma^{-1}\left(a_{k} U_{k}\right)\right] \sigma^{-1}\left(a_{k+1} U_{k+1}\right)+a_{k} U_{k}\left[F, \delta_{\sigma} \sigma^{-1}\left(a_{k+1} U_{k+1}\right)\right],
\end{aligned}
$$

one can see that $\zeta_{k}^{\sigma}-\zeta_{k+1}^{\sigma}=b \eta_{k}^{\sigma}$.

Lemma 5.6. 10] The cochain $\Psi_{D, \sigma}-\frac{1}{n}\left(\zeta_{1}^{\sigma}+\cdots+\zeta_{n}^{\sigma}\right)$ is a Hochschild coboundary.

Proof. Let $a_{0} U_{0}, a_{1} U_{1}, \ldots, a_{n} U_{n} \in \mathcal{A}_{G}$. Since

$$
\begin{aligned}
& |D|^{-1}\left[D, a_{j} U_{j}\right]_{\sigma}-\left[D, \sigma^{-1}\left(a_{j} U_{j}\right)\right]_{\sigma}|D|^{-1} \\
= & |D|^{-1}\left[D, a_{j}\right] U_{j}-\mu\left(U_{j}\right)\left[D, a_{j}\right] U_{j}|D|^{-1} \\
= & |D|^{-1}\left[D, a_{j}\right] U_{j}-\left[D, a_{j}\right]|D|^{-1} U_{j} \\
= & -|D|^{-1} \delta([D, a])|D|^{-1} U,
\end{aligned}
$$


in the expression for $\Psi_{D, \sigma}$ we can replace each $\left[D, \sigma^{-1}\left(a_{j} U_{j}\right)\right]_{\sigma}|D|^{-1}$ by $|D|^{-1}\left[D, a_{j} U_{j}\right]_{\sigma}$. Therefore

$$
\begin{aligned}
& \Psi_{D, \sigma}\left(a_{0} U_{0}, a_{1} U_{1}, \ldots, a_{n} U_{n}\right) \\
= & \operatorname{Tr}_{\omega}\left(\gamma a_{0} U_{0}\left[D, \sigma^{-1}\left(a_{1} U_{1}\right)\right]_{\sigma} \cdots\left[D, \sigma^{-n}\left(a_{n} U_{n}\right)\right]_{\sigma}|D|^{-n}\right) \\
= & \operatorname{Tr}_{\omega}\left(\gamma a_{0} U_{0}\left[D, \sigma^{-1}\left(a_{1} U_{1}\right)\right]_{\sigma}|D|^{-1} \cdots\left[D, \sigma^{-1}\left(a_{n} U_{n}\right)\right]_{\sigma}|D|^{-1}\right) .
\end{aligned}
$$

Also we have

$$
\left[D, \sigma^{-1}\left(a_{j} U_{j}\right)\right]_{\sigma}|D|^{-1}=\left[F, a_{j} U_{j}\right]+\delta_{\sigma} \sigma^{-1}\left(a_{j} U_{j}\right) D^{-1}+\left[F, \delta_{\sigma} \sigma^{-1}\left(a_{j} U_{j}\right)\right]|D|^{-1} .
$$

Since

$$
\begin{aligned}
{\left[F, \delta_{\sigma} \sigma^{-1}\left(a_{j} U_{j}\right)\right] } & =\mu\left(U_{j}\right)\left[F, \delta\left(a_{j}\right) U_{j}\right] \\
& =\mu\left(U_{j}\right)\left[F, \delta\left(a_{j}\right)\right] U_{j} \\
& =\mu\left(U_{j}\right)|D|^{-1}\left(\delta\left(\left[D, a_{j}\right]\right)-\delta^{2}\left(a_{j}\right) F\right) U_{j}
\end{aligned}
$$

the terms containing $\left[F, \delta_{\sigma} \sigma^{-1}\left(a_{j} U_{j}\right)\right]|D|^{-1}$ yield trace class operators which vanish under the Dixmier trace. So we can replace each

$$
\left[D, \sigma^{-1}\left(a_{j} U_{j}\right)\right]_{\sigma}|D|^{-1}
$$

by

$$
\left[F, a_{j} U_{j}\right]+\delta_{\sigma} \sigma^{-1}\left(a_{j} U_{j}\right) D^{-1} .
$$

Therefore (5.7) is the sum of $2^{n}$ terms. The term

$$
\operatorname{Tr}_{\omega}\left(\gamma a_{0} U_{0}\left[F, a_{1} U_{1}\right] \cdots\left[F, a_{n} U_{n}\right]\right)
$$

is zero because one can write $a_{0} U_{0}=F\left[F, a_{0} U_{0}\right]+F a_{0} U_{0} F$, and

$$
\gamma F\left[F, a_{0} U_{0}\right]\left[F, a_{1} U_{1}\right] \cdots\left[F, a_{n} U_{n}\right]
$$

is trace class. Therefore the term is equal to

$$
\begin{aligned}
& \operatorname{Tr}_{\omega}\left(\gamma F a_{0} U_{0} F\left[F, a_{1} U_{1}\right] \cdots\left[F, a_{n} U_{n}\right]\right) \\
= & (-1)^{n} \operatorname{Tr}_{\omega}\left(\gamma F a_{0} U_{0}\left[F, a_{1} U_{1}\right] \cdots\left[F, a_{n} U_{n}\right] F\right) \\
= & -\operatorname{Tr}_{\omega}\left(F \gamma a_{0} U_{0}\left[F, a_{1} U_{1}\right] \cdots\left[F, a_{n} U_{n}\right] F\right) \\
= & -\operatorname{Tr}_{\omega}\left(\gamma a_{0} U_{0}\left[F, a_{1} U_{1}\right] \cdots\left[F, a_{n} U_{n}\right]\right) .
\end{aligned}
$$

Hence it has to be zero.

The terms having exactly one factor of the form $\delta_{\sigma} \sigma^{-1}\left(a_{j} U_{j}\right) D^{-1}$ add up to $\frac{1}{n}\left(\zeta_{1}^{\sigma}+\cdots+\zeta_{n}^{\sigma}\right)$, and to finish the proof we show that the terms with more than one factors of the form $\delta_{\sigma} \sigma^{-1}\left(a_{j} U_{j}\right) D^{-1}$ are Hochschild coboundaries. For example let us consider the case when two consecutive factors of the above form 
yield the term

$$
\begin{aligned}
& \operatorname{Tr}_{\omega}\left(\gamma a_{0} U_{0}\left[F, a_{1} U_{1}\right] \cdots\left[F, a_{j-1} U_{j-1}\right] \delta_{\sigma} \sigma^{-1}\left(a_{j} U_{j}\right)\right. \\
& \left.D^{-1} \delta_{\sigma} \sigma^{-1}\left(a_{j+1} U_{j+1}\right) D^{-1}\left[F, a_{j+2} U_{j+2}\right] \cdots\left[F, a_{n} U_{n}\right]\right) \\
= & \operatorname{Tr}_{\omega}\left(\gamma a_{0} U_{0}\left[F, a_{1} U_{1}\right] \cdots\left[F, a_{j-1} U_{j-1}\right] \delta_{\sigma} \sigma^{-1}\left(a_{j} U_{j}\right)\right. \\
& \left.\delta_{\sigma} \sigma^{-2}\left(a_{j+1} U_{j+1}\right) D^{-2}\left[F, a_{j+2} U_{j+2}\right] \cdots\left[F, a_{n} U_{n}\right]\right) \\
= & \operatorname{Tr}_{\omega}\left(\gamma a_{0} U_{0}\left[F, a_{1} U_{1}\right] \cdots\left[F, a_{j-1} U_{j-1}\right] \delta_{\sigma} \sigma^{-1}\left(a_{j} U_{j}\right)\right. \\
& \left.\delta_{\sigma} \sigma^{-2}\left(a_{j+1} U_{j+1}\right)\left[F, \sigma^{-2}\left(a_{j+2} U_{j+2}\right)\right] \cdots\left[F, \sigma^{-2}\left(a_{n} U_{n}\right)\right] D^{-2}\right) .
\end{aligned}
$$

Now using the identity

$\delta_{\sigma}^{2} \sigma^{-2}\left(a_{j} a_{j+1}\right)=\delta_{\sigma}^{2} \sigma^{-2}\left(a_{j}\right) \sigma^{-2}\left(a_{j+1}\right)+2 \delta_{\sigma} \sigma^{-1}\left(a_{j}\right) \delta_{\sigma} \sigma^{-2}\left(a_{j+1}\right)+a_{j} \delta_{\sigma}^{2} \sigma^{-2}\left(a_{j+1}\right)$,

one can see that (5.8) is equal to $b \varphi_{j}^{\sigma}\left(a_{0} U_{0}, a_{1} U_{1}, \ldots, a_{n} U_{n}\right)$ where

$$
\begin{aligned}
& \varphi_{j}^{\sigma}\left(a_{0} U_{0}, a_{1} U_{1}, \ldots, a_{n-1} U_{n-1}\right) \\
= & \frac{(-1)^{j}}{2} \operatorname{Tr}_{\omega}\left(\gamma a_{0} U_{0}\left[F, a_{1} U_{1}\right] \cdots\left[F, a_{j-1} U_{j-1}\right]\right. \\
& \left.\delta_{\sigma}^{2} \sigma^{-2}\left(a_{j} U_{j}\right)\left[F, \sigma^{-2}\left(a_{j+1} U_{j+1}\right)\right] \cdots\left[F, \sigma^{-2}\left(a_{n-1} U_{n-1}\right)\right] D^{-2}\right) .
\end{aligned}
$$

Hence, considering (5.5), Condition 5.2 and (5.6) we have proved the following:

Theorem 5.7. 10 The cyclic cocycle $\Phi_{F}$ and the local Hochschild cocycle $2 \Psi_{D, \sigma}$ yield the same value on any Hochschild $n$-cycle

$$
c=\sum_{j=1}^{k} a_{0 j} U_{0 j} \otimes a_{1 j} U_{1 j} \otimes \cdots \otimes a_{n j} U_{n j}
$$

that satisfies Condition 5.2.

\section{A local index formula for twisted spectral triples}

In [17, an Ansatz for a local index formula for twisted spectral triples is given and its validity for twisted spectral triples obtained from scaling automorphisms of spectral triple, has been verified. This formula is given in terms of residue functionals and twisted commutators. In this section we sketch very briefly some of the ideas in [17].

\subsection{Moscovici's Ansatz}

In Subsection 4.4, we explained the heuristic manner for obtaining the local Hochschild cocycle for twisted spectral triples from the one for ordinary spectral 
triples. In a rather similar manner one can obtain Moscovici's Ansatz for a local index formula for twisted spectral triples from the original local index formula of Connes and Moscovici discussed in Section 2. The Ansatz suggests that for a $\sigma$-spectral triple $(\mathcal{A}, \mathcal{H}, D)$, the twisted version of the local character should be given by

$$
\begin{aligned}
& \varphi_{n}^{\sigma}\left(a_{0}, \ldots, a_{n}\right):= \\
& \sum_{k} c_{n, k} f a_{0}\left[D, \sigma^{-2 k_{1}-1}\left(a_{1}\right)\right]_{\sigma}^{\left(k_{1}\right)} \ldots\left[D, \sigma^{-2\left(k_{1}+\cdots+k_{n}\right)-n}\left(a_{n}\right)\right]_{\sigma}^{\left(k_{n}\right)}|D|^{-n-2|k|},
\end{aligned}
$$

where the iterated twisted commutators $[D, a]_{\sigma}^{(k)}$ are defined in [17]. Here again

$$
f P=\operatorname{Res}_{z=0} \operatorname{Trace}\left(P|D|^{-2 z}\right) .
$$

Therefore an analogue of the simple dimension spectrum hypothesis is assumed. Namely, one needs to assume that there exists a discrete subset of the complex plane such that for any operator $P$ in an algebra of twisted pseudodifferential operators associated to the twisted spectral triple $(\mathcal{A}, \mathcal{H}, D, \sigma), P|D|^{-2 z}$ is a trace class operator provided $\operatorname{Re}(z)$ is large enough, and $\zeta_{P}(z):=\operatorname{Trace}\left(P|D|^{-2 z}\right)$ has a meromorphic extension to the plane with at most simple poles in this discrete set. We note that, as it is emphasized in [17, there is no canonical way of constructing an algebra of twisted pseudodifferential operators for a general twisted spectral triple. Also, in the twisted case, for formulating analogue of the regularity condition, one needs to postulate that the automorphism $\sigma$ has an extension to a larger algebra which contains the twisted differential forms. Another necessary condition for the validity of the Ansatz is a $\sigma$-invariance of the residue functionals which is related to the Selberg principle for reductive Lie groups [17.

\subsection{A local index formula for spectral triples twisted by scaling automorphisms}

Let $\left(\mathcal{A}_{G}, \mathcal{H}, D\right)$ denote a twisted spectral triple obtained by scaling automorphisms of an ordinary finitely summable regular spectral triple, introduced in Subsection 3.3 and let $\Psi\left(\mathcal{A}_{G}, \mathcal{H}, D\right):=\Psi(\mathcal{A}, \mathcal{H}, D) \rtimes G$ denote the associated algebra of twisted pseudodifferential operators. The automorphism $\sigma$ extends to an automorphism of $\Psi\left(\mathcal{A}_{G}, \mathcal{H}, D\right)$ and it is required that for any $P$ in this algebra [17]:

$$
f P=f \sigma(P)
$$

Definition 6.1. [17] The twisted JLO bracket of order $q$ as a $q+1$-linear form on $\Psi\left(\mathcal{A}_{G}, \mathcal{H}, D\right)$ is defined by

$$
\begin{gathered}
\left\langle a_{0} U_{0}^{*}, \ldots, a_{q} U_{q}^{*}\right\rangle_{D}=\int_{\Delta_{q}} \operatorname{Trace}\left(\gamma a_{0} U_{0}^{*} e^{-s_{0} \mu\left(U_{0}\right)^{2} D^{2}} a_{1} U_{1}^{*} e^{-s_{1} \mu\left(U_{0} U_{1}\right)^{2} D^{2}} \ldots\right. \\
\left.\cdots a_{q} U_{q}^{*} e^{-s_{q} \mu\left(U_{0} \cdots U_{q}\right)^{2} D^{2}}\right)
\end{gathered}
$$


for all $a_{0}, \ldots, a_{q} \in \mathcal{A}$ and $U_{0}, \ldots, U_{q} \in G$, where

$$
\Delta_{q}:=\left\{s=\left(s_{0}, \ldots, s_{q}\right) \in \mathbb{R}^{q+1} ; \quad s_{j} \geq 0, \quad s_{0}+\cdots+s_{q}=1\right\} .
$$

In [17, it is assumed that $a_{0}, \ldots, a_{q}$ are polynomials in $D$ and elements of $\mathcal{A}$ and $[D, \mathcal{A}]$ which are homogeneous in $\lambda$ as $D$ is replaced by $\lambda D$. The expression obtained from replacing each $D$ occurring in $a_{0}, \ldots, a_{q}$ by $\epsilon^{1 / 2} D$ is denoted by $\left\langle a_{0} U_{0}^{*}, \ldots, a_{q} U_{q}^{*}\right\rangle_{D}(\epsilon)$. Equivalently, one has

$$
\left\langle a_{0} U_{0}^{*}, \ldots, a_{q} U_{q}^{*}\right\rangle_{D}(\epsilon)=\epsilon^{\frac{m}{2}}\left\langle a_{0} U_{0}^{*}, \ldots, a_{q} U_{q}^{*}\right\rangle_{\epsilon^{1 / 2} D},
$$

where $m$ is the total degree of $\lambda$ in $a_{0} \cdots a_{q}$ after replacing each $D$ by $\lambda D$.

Proposition 6.2. 17. If $a_{0} \in \mathcal{A}$ and $a_{1}, \ldots, a_{q} \in[D, \mathcal{A}]$, then there is an asymptotic expansion

$$
\left\langle a_{0} U_{0}^{*}, \ldots, a_{q} U_{q}^{*}\right\rangle_{D}(\epsilon) \sim \sum_{j \in J}\left(c_{j}+c_{j}^{\prime} \log \epsilon\right) \epsilon^{\frac{q}{2}-\rho_{j}}+O(1) \text { as } \epsilon \searrow 0
$$

where $\rho_{0}, \ldots, \rho_{m}$ are points in the half plane $\operatorname{Re}(z) \geq \frac{q}{2}$.

The twisted version of the JLO cocycles is defined by

$$
J^{q}(D)\left(A_{0}, \ldots, A_{q}\right)=\left\langle A_{0},\left[D, \sigma^{-1}\left(A_{1}\right)\right]_{\sigma}, \ldots,\left[D, \sigma^{-q}\left(A_{q}\right)\right]_{\sigma}\right\rangle_{D}
$$

for $A_{0}, \ldots, A_{q} \in \mathcal{A}_{G}$. Since in the twisted case this does not define a cocycle, in [17, by considering

$$
J^{q}\left(\epsilon^{1 / 2} D\right)\left(A_{0}, \ldots, A_{q}\right)=\epsilon^{\frac{q}{2}}\left\langle A_{0},\left[D, \sigma^{-1}\left(A_{1}\right)\right]_{\sigma}, \ldots,\left[D, \sigma^{-q}\left(A_{q}\right)\right]_{\sigma}\right\rangle_{\epsilon^{1 / 2} D}
$$

for $\epsilon>0$, and passing to the constant term using the above proposition, $\varphi_{q}^{\sigma}$ is defined by

$$
\varphi_{q}^{\sigma}\left(A_{0}, \ldots, A_{q}\right):=\left.\left\langle A_{0},\left[D, \sigma^{-1}\left(A_{1}\right)\right]_{\sigma}, \ldots,\left[D, \sigma^{-q}\left(A_{q}\right)\right]_{\sigma}\right\rangle_{D}\right|_{0} .
$$

It follows from the following two results [17 that for any twisted spectral triple obtained by conformal perturbation of an ordinary finitely summable regular spectral triple and satisfying a Selberg type invariance condition (6.9), one can associate a local cyclic cocycle for its Connes-Chern character.

Theorem 6.3. 17] The cochain $\left\{\varphi_{q}^{\sigma}\right\}$ satisfies the cocycle identity in the (b, B)-bicomplex:

$$
b \varphi_{q-1}^{\sigma}\left(a_{0} U_{0}^{*}, \ldots, a_{q} U_{q}^{*}\right)+B \varphi_{q+1}^{\sigma}\left(a_{0} U_{0}^{*}, \ldots, a_{q} U_{q}^{*}\right)=0,
$$

for all $a_{0}, \ldots, a_{q} \in \mathcal{A}$ and $U_{0}, \ldots, U_{q} \in G$.

Theorem 6.4. 17] The cocycle $\left\{\varphi_{q}^{\sigma}\right\}$ is cohomologous to the Connes-Chern character associated to the twisted spectral triple $\left(\mathcal{A}_{G}, \mathcal{H}, D\right)$ in the periodic cyclic cohomology $H P^{*}\left(\mathcal{A}_{G}\right)$. 


\section{References}

[1] M. Adler, On a trace functional for formal pseudo differential operators and the symplectic structure of the Korteweg-de Vries type equations. Invent. Math. 50, no. 3, 219-248, 1978/79.

[2] J.-B. Bost, Principe d'Oka, K-théorie et systèmes dynamiques non commutatifs, Invent. math., 101, 261-333, 1990.

[3] A. Connes, Noncommutative differential geometry, Inst. Hautes Études Sci. Publ. Math. No. 62, 257-360, 1985.

[4] A. Connes, The action functional in noncommutative geometry. Comm. Math. Phys. 117, no. 4, 673-683, 1988.

[5] A. Connes, Noncommutative Geometry. Academic Press, 1994.

[6] A. Connes, Noncommutative geometry year 2000, Highlights of mathematical physics (London, 2000), 49110, Amer. Math. Soc., Providence, RI, 2002.

[7] A. Connes and H. Moscovici, The local index formula in noncommutative geometry. Geom. Funct. Anal. 5, no. 2, 174-243, 1995.

[8] A. Connes and H. Moscovici, Type III and spectral triples. Traces in number theory, geometry and quantum fields, 57-71, Aspects Math., E38, Friedr. Vieweg, Wiesbaden, 2008.

[9] J. Dixmier, Existence de traces non normales. C. R. Acad. Sci. Paris Sér. A-B 262, A1107-A1108, 1966.

[10] F. Fathi Zadeh, Twisted Spectral Triples, Connes' Character Formula, and the Gauss-Bonnet Theorem for Noncommutative Two Tori, Ph.D. Thesis, 2010.

[11] F. Fathi Zadeh and M. Khalkhali, The Algebra of Formal Twisted Pseudodifferential Symbols and a Noncommutative Residue. Lett. Math. Phys. 94, no. 1, 41-61, 2010.

[12] J. M. Gracia-Bondia, J. C. Varilly, H. Figueroa, Elements of Noncommutative Geometry. Birkhäuser Advanced Texts, Birkhäuser Boston Inc., Boston, MA, 2001.

[13] N. Higson, The local index formula in noncommutative geometry. Contemporary developments in algebraic $K$-theory, 443-536 (electronic), ICTP Lect. Notes, XV, Abdus Salam Int. Cent. Theoret. Phys., Trieste, 2004.

[14] N. Higson, The residue index theorem of Connes and Moscovici. Surveys in noncommutative geometry, 71-126, Clay Math. Proc., 6, Amer. Math. Soc., Providence, RI, 2006. 
[15] Ch. Kassel, Le résidu non commutatif (d'après M. Wodzicki). (French) [The noncommutative residue (after M. Wodzicki)] Séminaire Bourbaki, Vol. 1988/89. Astérisque No. 177-178, Exp. No. 708, 199-229, 1989.

[16] Ju. I. Manin, Algebraic aspects of nonlinear differential equations. (Russian) Current problems in mathematics, Vol. 11 (Russian), pp. 5-152. (errata insert) Akad. Nauk SSSR Vsesojuz. Inst. Naučn. i Tehn. Informacii, Moscow, 1978.

[17] H. Moscovici, Local index formula and twisted spectral triples. Quanta of maths, 465-500, Clay Math. Proc., 11, Amer. Math. Soc., Providence, RI, 2010 .

[18] A. Polishchuk, Holomorphic bundles on 2-dimensional noncommutative toric orbifolds, Noncommutative geometry and number theory, 341-359, Aspects Math., E37, Vieweg, Wiesbaden, 2006.

[19] M. Wodzicki, Noncommutative residue. I. Fundamentals. K-theory, arithmetic and geometry (Moscow, 1984-1986). 320-399, Lecture Notes in Math., 1289, Springer, Berlin, 1987. 